\title{
A Systematic Review on Chemical Constituents of Suanzaoren Decoction, a Traditional Chinese Medicine Prescription
}

\author{
Wenchao Gu*, Tianyuan Ye*, Liangkun Zhang, Yong Yang, Dongmei Qi, \\ Xiaorui Cheng", Xin Wang\# \\ Shandong University of Traditional Chinese Medicine, Jinan, China \\ Email: "cxr916@163.com, "xinw0422@126.com
}

How to cite this paper: Gu, W.C., Ye, T.Y., Zhang, L.K., Yang, Y., Qi, D.M., Cheng, X.R. and Wang, X. (2021) A Systematic Review on Chemical Constituents of Suanzaoren Decoction, a Traditional Chinese Medicine Prescription. International Journal of Clinical Medicine, 12, 494-523. https://doi.org/10.4236/ijcm.2021.1211045

Received: October 11, 2021

Accepted: November 27, 2021

Published: November 30, 2021

Copyright $\odot 2021$ by author(s) and Scientific Research Publishing Inc. This work is licensed under the Creative Commons Attribution International License (CC BY 4.0).

http://creativecommons.org/licenses/by/4.0/

\begin{abstract}
Traditional Chinese Medicine prescription Suanzaoren decoction (SZRD) is composed of Ziziphi Spinosae Semen, Chuanxiong Rhizoma, Anemarrhenae rhizoma, Poria and Licorice. It was used to treat central nervous system diseases such as insomnia and anxiety for thousands of years. This paper aims to systematically understand varieties and quantities of compounds and clarify chemical components of SZRD, subsequently to further provide the reference for phytochemistry and pharmacology researches of SZRD. Our results showed that SZRD contained 145 components, including flavonoids, triterpenoids, steroids, coumarins, phthalides, and volatile oils, etc., while five-single herbs contain 1104 components. Only in terms of compound number, there were 80 common components in SZRD and its five herbs, which accounted for $6.8 \%$ of total compounds in all 5 herbs and $55.2 \%$ of compounds in SZRD. The components of SZRD were not simply the sum of one in every single herb. It is necessary to perform parallel studies among SZRD and its herbs. This review discussed the problems that existed in the chemical research of SZRD and pointed out the direction for its further research.
\end{abstract}

\section{Keywords}

Traditional Chinese Medicine, Suanzaoren Decoction, Chemical Ingredient

\section{Introduction}

Suanzaoren decoction (SZRD), a classic Traditional Chinese Medicine (TCM) prescription, was first described in the book "Jin Gui Yao Lue" by Zhongjing

${ }^{\star}$ Co-first authors, these two authors contributed equally to this work.

\#Corresponding author. 
Zhang in Han dynasty. SZRD is consists of five herbs, Suanzaoren (Ziziphi Spinosae Semen, Ziziphus jujuba Mill. var. spinosa (Bunge) Hu ex H.F. Chou), Chuanxiong (Chuanxiong Rhizoma, Ligusticum chuanxiong Hort.), Zhimu (Anemarrhenae rhizoma, Anemarrhena asphodeloides Bge.), Fuling (Poria, Poria cocos (Schw.) Wolf) and Gancao (Licorice, Glycyrrhiza uralensis Fisch., Glycyrrhiza inflata Bat. or Glycyrrhiza glabra L.) at a ratio of 15:6:6:6:3. In the clinic of TCM, SZRD is the most common herbal formula prescribed by TCM doctors to treat central nervous system diseases such as insomnia [1] [2] [3], secondary insomnia [4] [5] [6] [7], anxiety [8], depression [9], menopausal syndrome [10] and other diseases [11] [12]. Clinical researches showed that the combination of SZRD and other drugs, including TCM and chemicals, can improve insomnia [13] [14] [15] [16], anxiety [16] [17] [18], depression [19] [20] and other diseases [21], enhance the efficiency of treatment, and reduce adverse reactions. Besides, SZRD has pharmacological characteristics of immune protective and sedative effects [22].

The clinical therapeutic effect and pharmacological function of SZRD originate in its chemical composition. A vast lot of chemical components in SZRD have been isolated and identified using high-performance liquid chromatography (HPLC) and mass spectrometry (MS) technology. In this paper, we investigated and analyzed chemical ingredients of SZRD and its five-single herbs on basis of literatures in the last 5 years and calculated the percentage based on compounds number of a certain class in the total number of compounds. We also focused on similarities and differences of ingredients between SZRD and its herbs.

\section{Chemical Constituents of Five Herbs in Suanzaoren Decoction}

\subsection{Suanzaoren (Ziziphi Spinosae Semen, Ziziphus jujuba Mill. var. spinosa (Bunge) Hu ex H.F. Chou)}

We used "Suanzaoren”, “Zaoren”, “Ziziphi Spinosae Semen”, “Jujube”, “Ziziphus jujuba Mill. var. spinosa (Bunge) Hu ex H. F. Chou" as key words to search chemical components of Ziziphi Spinosae Semen. We mainly focused on the literatures of the past five years, and tried to get more ingredients at the same time. Results showed mounts of literatures on ingredients of Ziziphi Spinosae Semen have been reported until now. The 36 chemical components in standard decoction of Ziziphi Spinosae Semen are isolated by using UPLC-Q/TOF-MS and UPLC-PDA system, including nucleosides, phenolic acids, alkaloids and flavonoids [23]. The 109 components are identified in Ziziphi Spinosae Semen by UPLC-Q/TOF-MS and principal component analysis (PCA), including 66 flavonoids, 15 triterpenoid saponin, 19 alkaloids, 8 terpenoid acids and 1 phenolic acid [24]. Research on components in crude and parched Ziziphi Spinosae Semen shows that 40 chemical ingredients (flavonoids, saponins, alkaloids and triterpenoids, etc.) are changed during stir-frying process and 19 key markers 
can contribute to classification of crude and parched Ziziphi Spinosae Semen [25]. The sedative and hypnotic effects of Ziziphi Spinosae Semen were significantly enhanced after processing. These researches provided a rapid and effective approach to monitor quality consistency of Ziziphi Spinosae Semen.

We totally collected 175 compounds in Ziziphi Spinosae Semen [23]-[40], which were divided into 8 categories, including flavonoids (62 compounds), alkaloids (28 compounds), amino acids (6 compounds), nucleosides (4 compounds), phenolic acids (4 compounds), triterpenoids (61 compounds), volatile oils (9 compounds) and others (1 compound) (Table 1). Ziziphi Spinosae Semen has the highest proportion of flavonoids (36\%), followed by triterpenes (35\%), and the lowest proportion of phenolic acids (2\%) and nucleotides (2\%) (Figure $1(\mathrm{a}))$.

\subsection{Chuanxiong (Chuanxiong Rhizoma, Ligusticum chuanxiong Hort.)}

The chemical constituents of Chuanxiong Rhizoma are searched by using "Chuanxiong", "Chuanxiong Rhizoma", "Ligusticum chuanxiong Hort.", "Ligusticum sinense Oliv" and we focused on the literature of the past five years, including English and Chinese literature. The study indicated that 73 chemical compounds in the essential oil from Chuanxiong Rhizoma were detected and 33 compounds with main component Z-Ligustilide were identified by gas chromatgraphy-mass spectrometry (GC-MS) [41]. The other study showed that 30 chemical components in Chuanxiong Rhizoma were separated and identified by using ultra-performance liquid chromatography with electrospray ionizationtime of flight mass spectrometry (UPLC-ESI-TOF/MS) technology, including 20 phthalides, 2 flavonoids, 1 alkaloid and 7 phenolic acids [42]. Research on components of dried rhizome from Chuanxiong Rhizoma showed that 10 phthalide derivatives were isolated by using HPLC technology and spectroscopic analyses and 6 of them were identified as ligusticoside A-F for the first time [43]

Chuanxiong Rhizoma contained 324 compounds, which could be divided into 10 categories [41]-[56], including volatile oils (162 compounds, 50\%), phthalides (chemical classification instead of plastic pollution) (85 compounds, 26\%), phenolic acids (20 compounds, $6 \%$ ), polysaccharides (17 compounds, 5\%), alkaloids (14 compounds, $4 \%$ ), flavonoids (6 compounds, $2 \%$ ), ceramides and cerebrosides (5 compounds, $2 \%$ ), coumarins ( 3 compounds, $1 \%$ ), triterpenoids ( 4 compounds, $1 \%$ ) and others (8 compounds, $3 \%$ ) (Table 2, Figure 1(b)).

\subsection{Zhimu (Anemarrhenae rhizoma, Anemarrhena asphodeloides Bge.)}

We used "Zhimu", "Anemarrhenae rhizoma", "Anemarrhena asphodeloides Bge.", "Anemarrhena asphodeloides Bunge" as key words to search chemical components of Anemarrhenae rhizoma, and the literatures were mainly in recent years which contained all the components as much as possible. It's reported that 32 chemical constituents were identified in Anemarrhenae rhizoma, which 


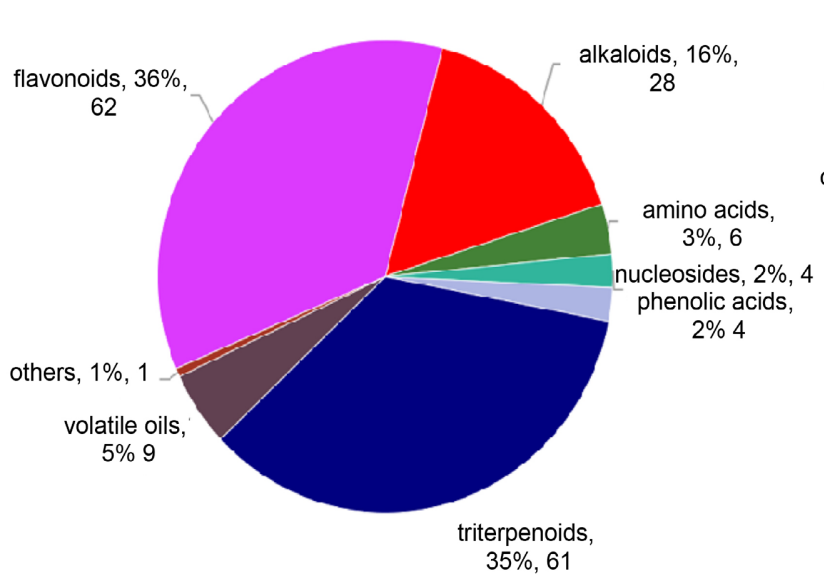

(a)

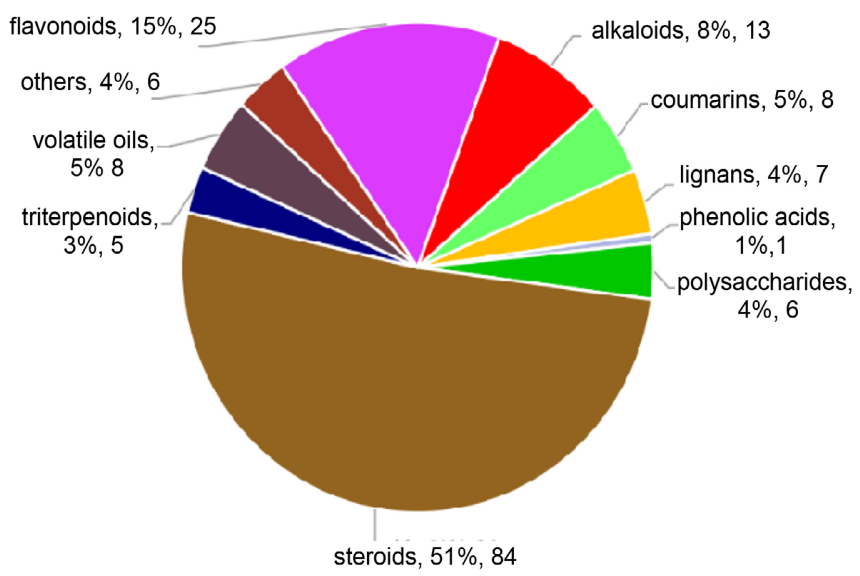

(c)

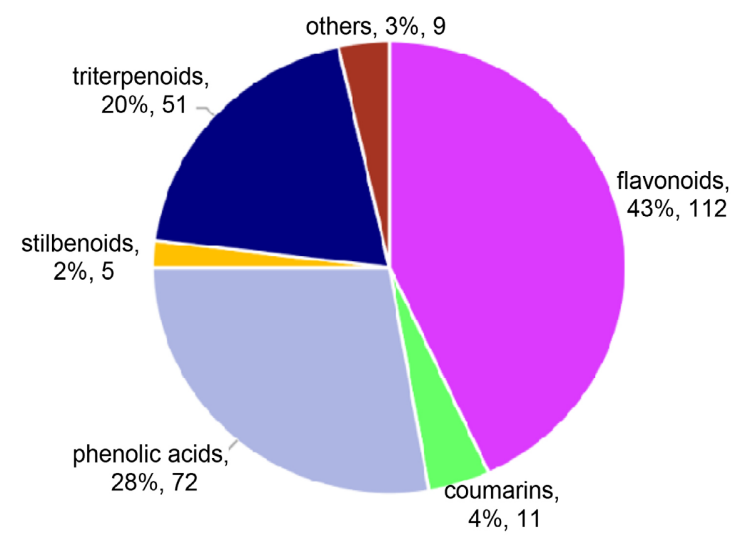

(e)

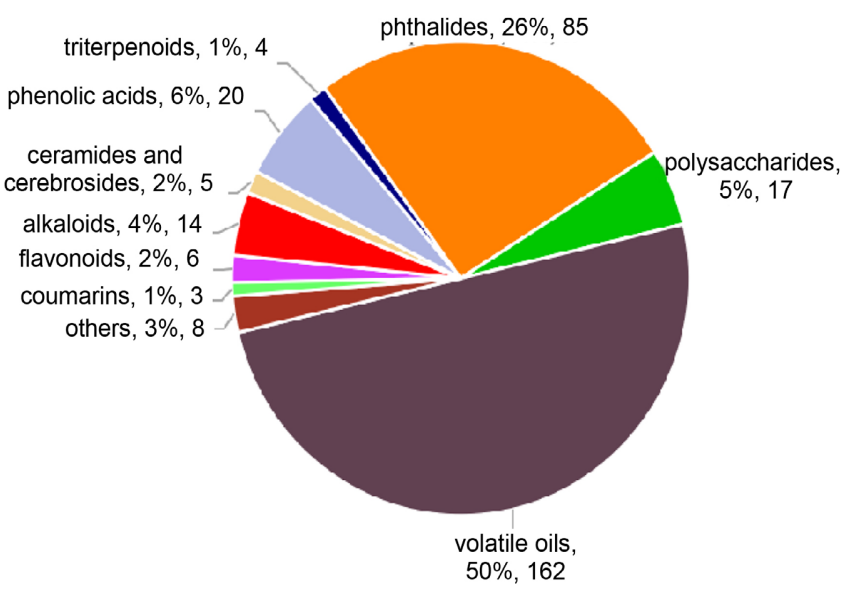

(b)

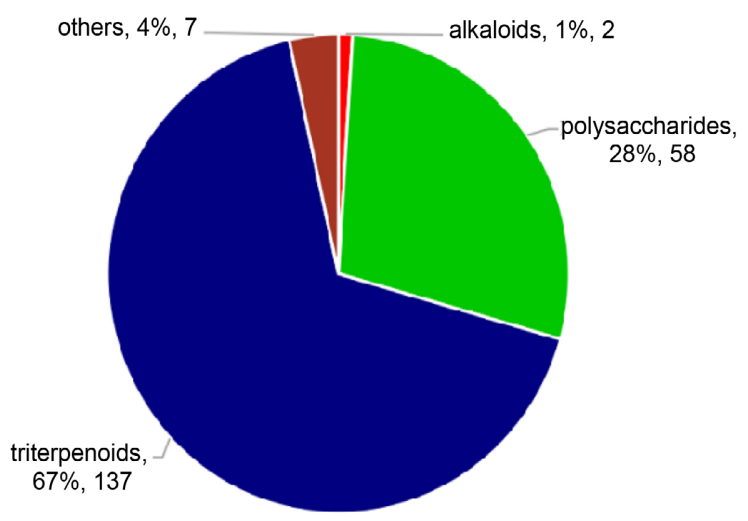

(d)

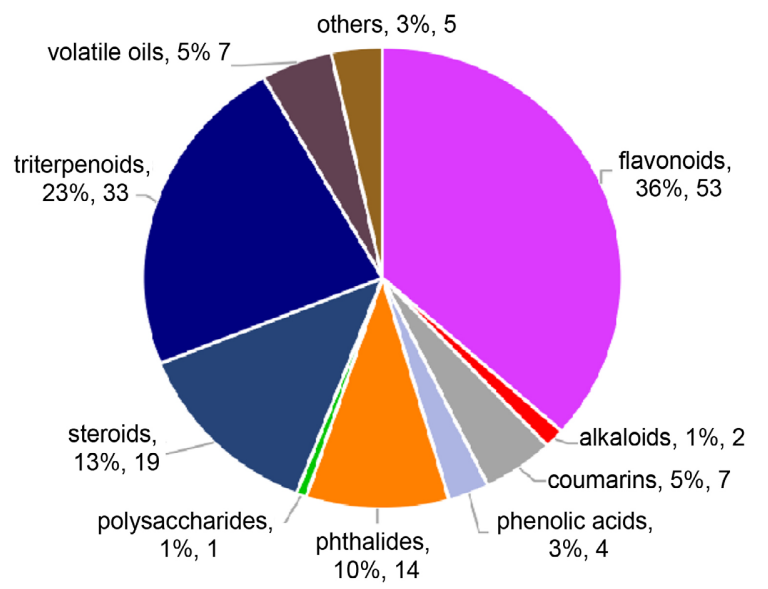

(f)

Figure 1. Chemical constituents in suanzaoren decoction and its herbs. (a) Constituents in Ziziphi Spinosae Semen; (b) Constituents in Chuanxiong Rhizoma; (c) Constituents in Anemarrhenae rhizome; (d) Constituents in Poria; (e) Constituents in Licorice; (f) Constituents in SZRD. 
Table 1. Chemical constituents in Suanzaoren (Ziziphi Spinosae Semen, Ziziphus jujuba Mill. var. spinosa (Bunge) Hu ex H. F. Chou).

\begin{tabular}{|c|c|}
\hline Class & Compound \\
\hline Triterpenoids & $\begin{array}{l}\text { 24-hydroxyceanothic acid, 2-o-protocatechuoyl aliphitolic acid, } 2 \alpha \text {-hydroxypyracrenic acid, 3-ketoursolic } \\
\text { acid, } 5 \alpha, 8 \alpha \text {-epidioxy-(22e,4r)-ergosta-6,22-dien-3 } \beta \text {-ol, acetyl jujuboside B, alhpitolic acid, betulic acid, } \\
\text { betulin, betulinic acid, betutin, campesterol, ceanothenic acid, ceanothic acid, epiceanothic acid, jujuboside } \\
\text { A, jujuboside } \mathrm{A}_{1} \text {, jujuboside } \mathrm{A}_{2} \text {, jujuboside } \mathrm{B} \text {, jujuboside } \mathrm{B}_{1} \text {, jujuboside c, jujuboside } \mathrm{D} \text {, jujuboside } \mathrm{E} \text {, } \\
\text { jujuboside } \mathrm{G} \text {, jujuboside h, jujuboside I, jujuboside II, jujuboside III, jujuboside IV, lupeol, methyl } \\
\text { betulinate, projujuboside B, protojujuboside A, protojujuboside B, protojujuboside } \mathrm{B}_{1} \text {, protojujuboside } \mathrm{D} \text {, } \\
\text { protojujuboside } \mathrm{H} \text {, pseudolaroside B, stigmast-4-en-3-one, ursolic acid, } 2 \alpha \text {-hydroxyursolic acid, } \\
\text { 3-o-cis-p-coumaroyl alphitolic acid, 3-o-cis-p-coumaroyl maslinic acid, 3-o-protocatechuoyl ceanothic acid, } \\
\text { 3-o-trans-p-coumaroyl alphitolic acid, 3-o-trans-p-coumaroyl maslinic acid, Azukisaponin II, betulonic } \\
\text { acid, cecropiacic acid, colubrinic acid, corosolic acid, hydroxyoleanonic acid lactone, isoceanothic acid, } \\
\text { lulutonic acid, maslinic aicd, oleanolic acid, pomonic acid, zizyberanalic acid, zizyphus saponin I, } \\
\text { zizyphus saponin II, zizyphus saponin III }\end{array}$ \\
\hline Flavonoids & 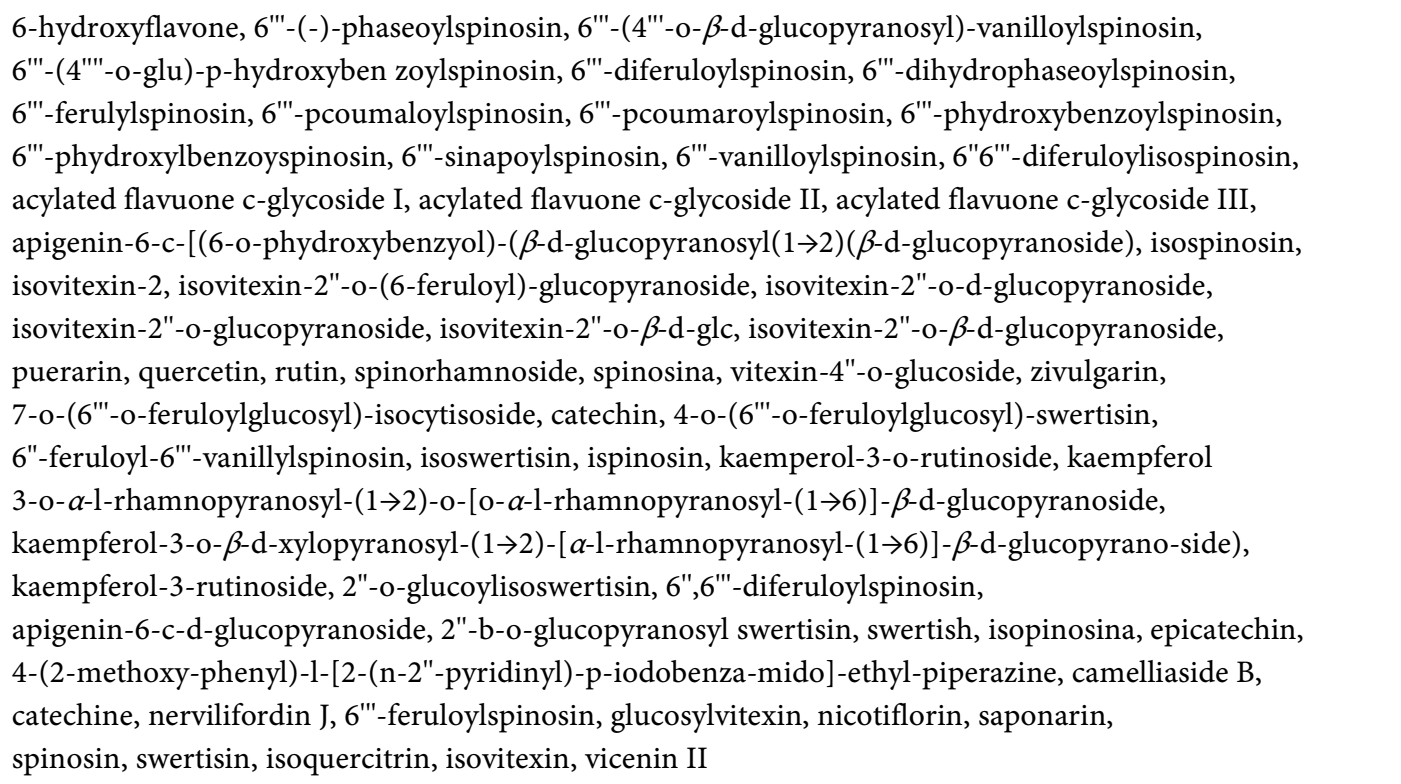 \\
\hline Alkaloids & $\begin{array}{l}\text { amphibine, coclaurine } K \text {, lysicamine, nornuciferine } I_{a} \text {, nornuciferine } I_{b} \text {, nuciferine e, sanjoinine } A \text {, } \\
\text { sanjoinine } B \text {, sanjoinine } C \text {, sanjoinine } D \text {, sanjoinine e, sanjoinine } F \text {, sanjoinine } G_{1} \text {, sanjoinine } G_{2} \text {, } \\
\text { sanjoinine } I_{a} \text { (nornuciferine), sanjoinine } I_{b} \text { (norisocorydine), zizyphusine, } \\
\text { 5-hydroxy-6-methoxynoraporphine, n-methylasimilobine, } \\
\text { 6-(2',3'-dihydroxyl-4'-hydroxymethyl-tetrahydro-furan-1'-yl)-cyclopentene[c]pyrrole-1,3-diol, jubanine } C \text {, } \\
\text { jubanine F, jujube cyclic peptide, juzirine, nummularine } B \text {, adenosine, magnoflorine, tryptophan }\end{array}$ \\
\hline Amino Acids & $\begin{array}{l}\text { aminocaproic acid, citric acid, cycol(arginine-proline), glutamic acid dipeptide, phenylalanine, } \\
\text { 6-glu-coclaurine }\end{array}$ \\
\hline Nucleosides & $\begin{array}{l}\text { cyclic adenosine monophosphate, cyclic guanosine monophosphate, guanosine hydrate, uridine } \\
\text { monophosphate }\end{array}$ \\
\hline Phenolic Acids & hydroxybenzoic acid, linoleic acid, protocatechuic acid, oleic acid \\
\hline Volatile Oils & $\begin{array}{l}\text { 9,12-octadecadienoic acid, 9-octadecenoic acid, arachidic acid, docosanoic acid, lauric acid, } \\
\text { myristic acid, palmitic acid, palmitoleic acid, stearic acid }\end{array}$ \\
\hline Others & ebelin lactone \\
\hline
\end{tabular}


Table 2. Chemical constituents in Chuanxiong (Chuanxiong Rhizoma, Ligusticum chuanxiong Hort.).

\begin{tabular}{|c|c|}
\hline Class & Compound \\
\hline Volatile Oils & 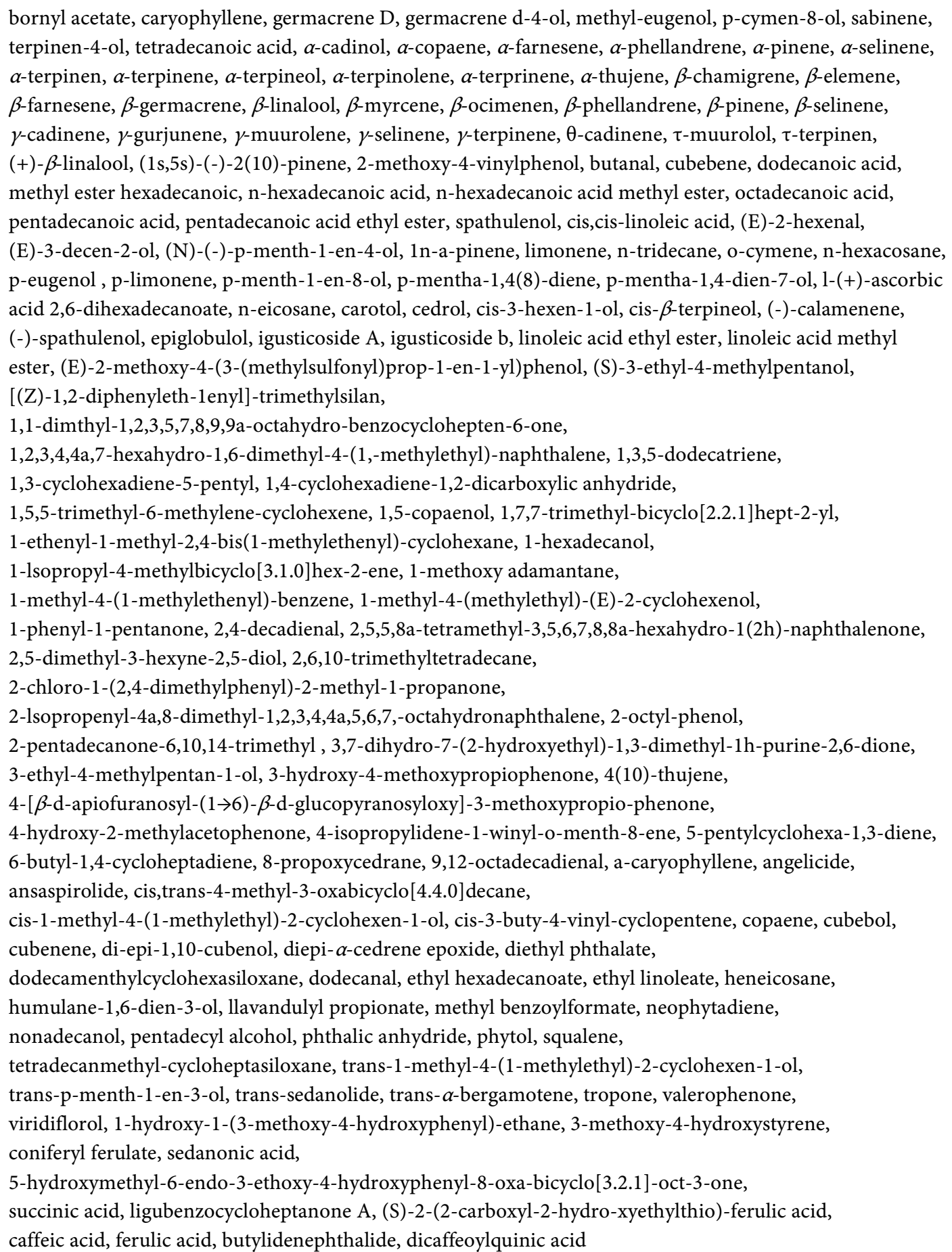 \\
\hline
\end{tabular}

1 -acetyl- $\beta$-carboline, 1- $\beta$-ethylacrylate-7-aldehydo- $\beta$-carboline,

Alkaloids

2,3-diphenyl-1,3,4-oxadiazol-3-ium-5-olaten-pentyl-4,5-dihydrophthalide, adenine, adenosine, choline, cyclotetradecane, ligustrazine, l-isobutyl-l-valine anhydride,

l-valine-l-valine anhydride, pelolyrine, tetramethylpyrazine, trimethylamine, uracil 


\section{Continued}

\begin{tabular}{|c|c|}
\hline $\begin{array}{l}\text { Ceramides and } \\
\text { Cerebrosides }\end{array}$ & $\begin{array}{l}\text { (2N)-2-hydroxy-n-[(2s,3s,4n,8e)-1,3,4-trihydroxypentadec-8-en-2-yl]heptacosanamide, } \\
(2 \mathrm{~N}) \text {-2-hydroxy-n-[(2s,3s,4s,8e)-1,3,4-trihydroxyicos-8-en-2-yl]tetracosanamide, } \\
(2 \mathrm{~N}) \text {-2-hydroxy-n- }(3 \mathrm{~s}, 4 \mathrm{~s}, 5 \mathrm{~s}) \text {-4-hydroxy-5-[(4e)-undec-4-en-1-yl]tetrahydrofuran-3-yl heptacosanamide, } \\
(2 \mathrm{~N}) \text {-n-[(2n,3s,4n,8e)-1-( } \beta \text {-d-glucopyranosyloxy)-3,4-dihydroxyoctadec-8-en-2-yl]-2-hydroxyhexadecanam } \\
\text { ide, } \\
(2 \mathrm{~N})-\mathrm{n} \text {-[(2s,3n,4e,8e)-1-( } \beta \text {-d-glucopyranosyloxy)-3-hydroxydodeca-4,8-dien-2-yl]-2-hydroxydocosanamide }\end{array}$ \\
\hline Coumarins & 5,7,8-trimethyl-dihydrocoumarin, scopoletin, cnidioside A \\
\hline Flavonoids & apigenin, apigenin-7-o- $\beta$-d-glueuronide, daidzein, astragalin, 2-(1-oxopentyl)-benzoic acid methyl ester \\
\hline Phenolic Acids & $\begin{array}{l}\text { icariside } \mathrm{F}_{2} \text {, ligusticumacid A, ligusticumacid B, ligusticumacid C, ligusticumacid d, ligusticumacid e, } \\
\text { ligusticumacid F, 5-hydroxymethyl-6-endo-3-methoxy-4-hydroxyphenyl-8-oxa-bicyclo (3.2.1)-oct-3-one, } \\
\text { chrysophanol, folic acid, gallic acid, palmitinic acid, p-hydroxybenzoic acid, sinapic acid, vanillin, } \\
\text { chlorogenic acid, vanillic acid, linoleic acid, protocatechuic acid, oleic acid }\end{array}$ \\
\hline
\end{tabular}

levistilide A, ligustilide, 3-butylene-4,5-dihydroxyph-thailde, senkyunolide $\mathrm{Q}$, senkyunolide $\mathrm{R}$, senkyunolide $\mathrm{S}$, e-senkyunolide $\mathrm{E}$, ligusticoside A, z-ligustilide , $\mathrm{z}$-senkyunolide $\mathrm{E}$, ( $\left.\mathrm{z}, \mathrm{z}^{\prime}\right)$-6,8',7,3'-diligustilide, 3-butylidene-6-hydroxy-5,6-dihydrophthalide, 3-carboxyethyl-phthalide, 4,5-dihydro-3-butylidenephthalide, 3,6,8,3a-diligustilide, 3,8-dihydrodiligustilide, 3-butylidenephthalide, 3-hydroxy-4,5,6,7-tetrahydro-6,7-dihydroxy-3-butylphthalide, 4,5-dihydro-3-butylphthalide, 4,7-dihydroxy-3-butylphthalide, 4-hydroxy-3-butylphthalide, butylphthalide, chuanxiongins A, chuanxiongins $B$, chuanxiongins $C$, chuanxiongins $d$, chuanxiongins $E$, chuanxiongins $F$, chuanxiongnolide $A$, chuanxiongnolide $B$, chuanxiongnolide $R_{1}$, chuanxiongnolide $R_{2}$, hexahydro-3-butylphthalide, levistolide A, neocnidilide, senkyunolide $B$, senkyunolide $C$, senkyunolide $E$, senkyunolide $G$, senkyunolide $K$,

Phthalides senkyunolide 1 , senkyunolide $M$, senkyunolide $N$, senkyunolide $O$, senkyunolide $P$, tokinolide $B$, wallichilide, z,z'-3,3',8,8'-diligustilide, z'-3,8-dihydro-6,6',7,3'a-diligustilide, z-6,7-epoxyligustilide, z-butylidenephthalide, z-ligustilide dimer e-232, 3-butylidene-7-hydroxyphthalide, 3-carboxyrthyl-phthalide, 6-hydroxy-senkyunolide B, celephthalide A, chuanxiongoside A, chuanxiongoside $B$, (3'z)-(3s,8n,3a's,6'n)-4,5-dehydro-3.3a',8.6'-diligustilide, (3'z)-(3s,8s,3a's,6'n)-4,5-dehydro-3.3a',8.6'-diligustilide, thiosenkyunolide C, trans-neocrididilide, chuanxiongdiolide $A$, chuanxiongdiolide $B$, chuanxiongdiolide $\mathrm{L}_{1}$, chuanxiongdiolide $\mathrm{L}_{2}$, chuanxiongdiolide $\mathrm{L}_{3}$, chuanxiongdiolide $\mathrm{L}_{4}$, chuanxiongdiolide $\mathrm{L}_{5}$, chuanxiongdiolide $\mathrm{R}_{1}$, chuanxiongdiolide $\mathrm{R}_{2}$, chuanxiongdiolide $\mathrm{R}_{3}$, igustilide, 3-butyl-4-hydroxyphthalide, 3-butylphthalide, e-ligustilide, riligustilide, senkyunolide A, senkyunolide D, senkyunolide F, senkyunolide h, senkyunolide I, senkyunolide J, z,z'-6,8',7,3'-diligustilide, cnidilide

Polysaccharides LCA, LCB, LCC, LCX0, LCX1, LCX2, LCP-1, LCP-2, LCP-3, LCP-4, Arabinose, galactose, galacturonic acid, glucose, glucuronic acid, nhamnose, mannose

Triterpenoids progesterone, xiongterpene, Globulol, ergosterol peroxide

(-)-alloaromadendrane- $4 \beta, 10 \alpha, 13,15$-tetrol, 3 -o- $\beta$-d-apiofuranosyl-( $1 \rightarrow 6)$ - $\beta$-d-glucopyranoside,

Others 4-pentylcyclohex-3-ene-1 $\alpha, 2 \beta$-diol, campest-4-en-3-one, monopalmitin, $\beta$-d-apiofuranosyl-( $1 \rightarrow 6)$ - $\beta$-d-glucopyranosyl-3,4-dimethoxy-benzoate, aurantiamide acetate, lignoceric acid

contained 18 steroids, 6 flavonoids, 4 phenylpropanoids, 2 alkaloids, and 2 benzophenones using UPLC-Q-TOF/MS combination with characteristic fragments filter and neutral loss filter method [57]. The study on chemical constitutions of crude Anemarrhenae rhizoma (CAR) and salt-processed Anemarrhenae rhizoma (SAR) showed that a total of 24 components as main contributors had significant difference, and 7 main constituents were simultaneously determined by 
ultra-high-performance liquid chromatography-quadrupole mass spectrometry (UHPLC-MS), timosaponin $\mathrm{N}$, timosaponin $\mathrm{E}_{1}$, timosaponin BII, timosaponin BIII, anemarrhenasaponin I, timosaponin AII and timosaponin AIII [58].

We sorted out 163 components of Anemarrhenae rhizoma [57]-[76], which were classified into 10 categories, including steroids (84 compounds, 51\%), flavonoids (25 compounds, 15\%), alkaloids (13 compounds, $8 \%$ ), coumarins (8 compounds, $5 \%$ ), volatile oils ( 8 compounds, $5 \%$ ), triterpenoids (5 compounds, $3 \%$ ), polysaccharides ( 6 compounds, $4 \%$ ), lignans (7 compounds, $4 \%$ ), phenolic acids ( 1 compound, $1 \%$ ) and others (6 compounds, $4 \%$ ) (Figure 1(c), Table 3 ).

\subsection{Fuling (Poria, Poria Cocos (Schw.) Wolf.)}

We used key words "Fuling", "Poria”, "Poria $\operatorname{cocos}$ (Schw.) Wolf.", “Wolfiporia extensa (Peck) Ginns" to find the ingredients in Poria, and selected literatures in the past five years. We found many methods have been developed for analysis and quality control of Poria. For instance, qualitative and quantitative methods to analyze carbohydrates (polysaccharides, oligosaccharides and monosaccharides) in three different parts (epidermis, middle and inner) of Poria by high performance gel permeation chromatography coupled with charged aerosol detector (HPGPC-CAD), altra-performance liquid chromatography coupled with triple quadrupole mass spectrometry (UHPLC-QqQ-MS/MS), PCA and orthogonal partial least squared discriminant analysis (OPLS-DA) [77]. It is an efficient dereplication strategy to identify triterpene acid analogues in Poria based on ultra-performance liquid chromatography with electrospray ionisation quadrupole time-of-flight tandem mass spectrometry (UPLC-ESI-QTOF-MS/MS), and 62 triterpene acids were characterized [78]. A method based on UHPLC-MS combined metabolomics approach is established to explain the distribution of triterpene compounds in four parts, which are Poriae Cutis (PC), Rubra Poria (RP), White Poria (WP) and Poria cum Radix Pini (PRP) and 51 triterpene compounds are tentatively identified in Poria. The PC and PRP show a quite clear discrimination by the PCA and OPLS-DA, and 12 differential compounds are found [79].

The four classes of chemical components in Poria included 204 compounds [77]-[96], containing triterpenoids (137 compounds, 67\%), polysaccharides (58 compounds, $28 \%$ ), alkaloids ( 2 compounds, $1 \%$ ) and others (7 compounds, $4 \%$ ) (Table 4, Figure 1(d)).

\subsection{Gancao (Licorice, Glycyrrhiza uralensis Fisch., Glycyrrhiza inflata Bat. or Glycyrrhiza Glabra L.)}

We used key words "Gancao", "Zhigancao", "Licorice”, "Glycorrhizae Radix et Rizoma”, "Glycorrhiza urensis Fisch.", "Glycorrhiza inflata Bat.", "Glycorrhiza glabra L." to find the ingredients in Licorice, and we were mainly based on the literature of the past five years and delete the literature with duplicate components. 
Table 3. Chemical constituents in Zhimu (Anemarrhenae rhizoma, Anemarrhena asphodeloides Bge.).

\begin{tabular}{|c|c|}
\hline Class & Compound \\
\hline Steroids & 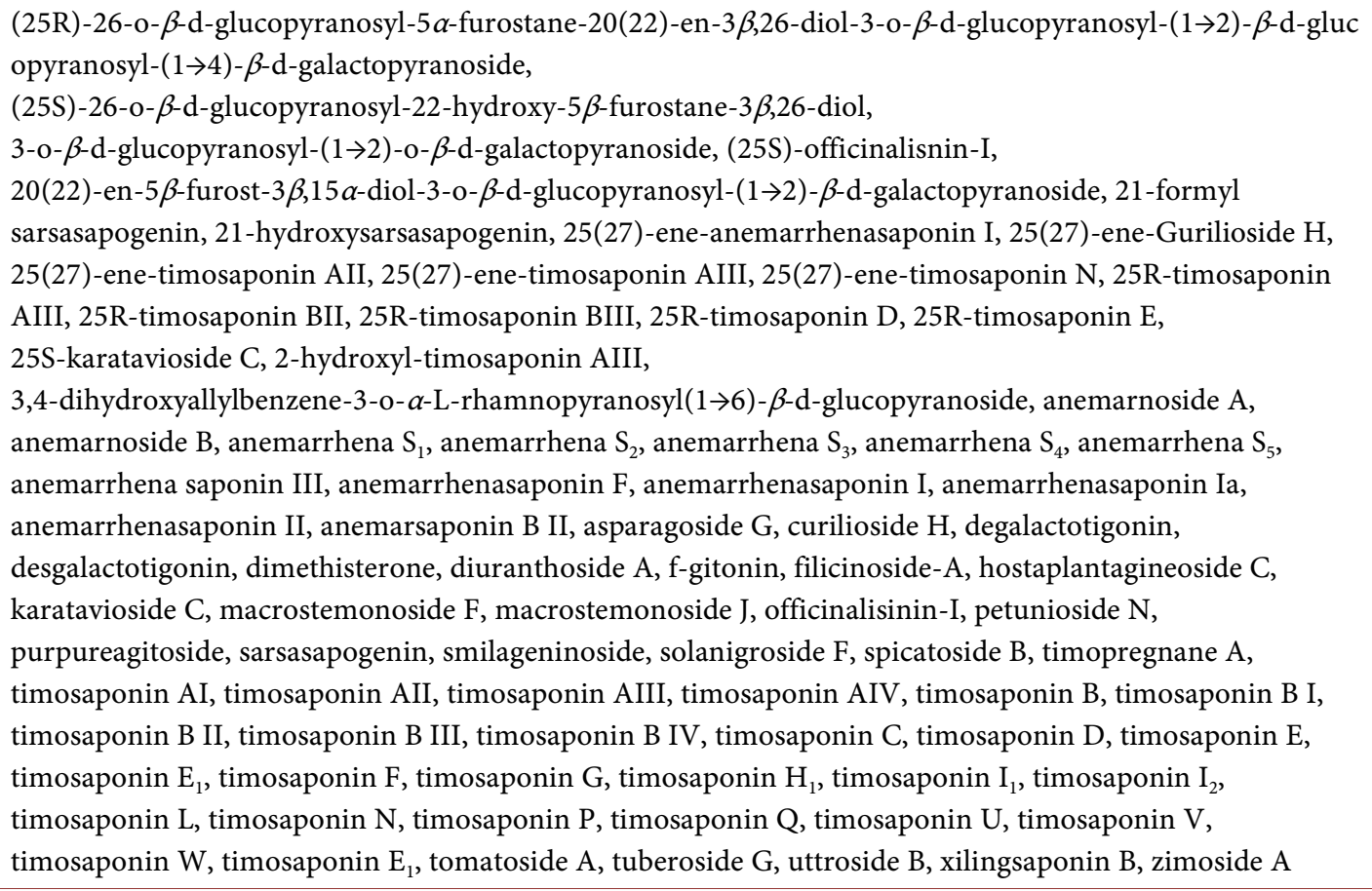 \\
\hline Alkaloids & $\begin{array}{l}\text { adenosine, coumaroyl tyramine, cyclo dileucyl, cyclo hetaleucyl, cyclo hexaleucyl, cyclo nonaleucyl, } \\
\text { cyclo octaleucyl, cyclo pentaleucyl, cyclo tetraleucyl, cyclo trileucyl, n-cis-feruloyl tyramine, } \\
\text { n-trans-feruloyltyramine, tryptophan }\end{array}$ \\
\hline Polysaccharides & d-galactose, d-galacturonic acid, d-glucose, d-mannose, 1-arabinose, 1-rhamnose \\
\hline Phenolic Acids & oleic acid \\
\hline Flavonois & $\begin{array}{l}\text { (2,6-dihydroxy-3-(((1s,4s,6r)-4-hydroxy3,7-dioxabicyclo[4-1.0]heptan-4-yl)methyl)-4-methoxyphenyl)(4-hy } \\
\text { droxyphenyl)methanone, (2s)-7,4'-dihydroxy-5-methoxyflavone, } \\
\text { (4-hydroxyphenyl)(2,3,4-trihydroxyphenyl)methanone, } \\
\text { (s)-5-(2,4-dihydroxy-3-(4-hydroxybenzoyl)-6-methoxyphenyl)pyrrolidin-2-one, } \\
\text { 2,4',6-trihydroxy-4-methoxy benzophenone, 2,6,4'-trihydroxy-4-methoxy Benzophenone, 2',4',4-trihydroxy } \\
\text { chalcone, 2'-o-methylisoliquiritigenin, 2'-o-methylphloretin, } \\
\text { 4',6-dihydroxy-4-methoxybenzophenone-2-o-(2"),3-c-(1")-1"-desoxy- } \alpha \text {-l-fructofuranoside, } \\
\text { anemarchalconyn, e-4'-demethyl-6-methyleucomin, } \\
\text { E-5,7-dihydroxy-3-(4'-hydroxybenzylidene)chroman-4-one, foliamangiferoside A, iriflophene, } \\
\text { iriflophenone, iriflpohenone-3-c- } \beta \text {-d-glucoside, isomangiferin, isosakuranetin, isovitexin, mangiferin, } \\
\text { mangiferoxanthone A, methyl 2-(2,4-dihydroxy-3-(4-hydroxybenzoyl)-6-methoxyphenyl) acetate, } \\
\text { neomangiferin, vitexin }\end{array}$ \\
\hline Triterpenoids & platycodin $\mathrm{D}$, platycodin $\mathrm{D}_{2}$, platycodin $\mathrm{D}_{3}$, platycoside $\mathrm{A}$, polygalacin $\mathrm{D}_{2}$ \\
\hline Volatile Oils & $\begin{array}{l}\text { dihydroxy-octadecadienoic acid, dihydroxy-octadecaenoic acid, dihydroxy-octadecatrienoic acid, } \\
\text { hydroxy-octadecadienoic acid, hydroxy-octadecatrienoic acid, inolenic acid, tearic acid, } \\
\text { trihydroxy-octadecaenoic acid }\end{array}$ \\
\hline Coumarins & $\begin{array}{l}\text { cis-hinokiresinol, hinokiresinol, methoxy-cis-hinokiresinol, monomethyl-cis-hinokiresinol, } \\
\text { oxy-hinokiresinol, trans-hinokiresinol, broussonin A, broussonin B }\end{array}$ \\
\hline Lignans & $\begin{array}{l}\text { 3"-hydroxy-4"-methoxy-4"-dehydroxynyasol, 3"-methoxynyasol, 4-hydroxy } \\
\text { acetophenone, 4-hydroxybenzaldehyde, 4'-o-methylnyasol, anemarcoumarin A, nyasol, }\end{array}$ \\
\hline Others & $\begin{array}{l}\text { (s)-5-(2-hydroxy-5-(3-(4-hydroxy phenyl) propyl)-4-methoxy phenyl) pyrrolidin-2-one, } \\
\text { aneglycoside A, aneglycoside B, aneglycoside C, phytosphingosine, } \alpha \text {-d-Glucose monoallyl ether }\end{array}$ \\
\hline
\end{tabular}


Table 4. Chemical constituents in Fuling (Poria, Poria cocos (Schw.) Wolf).

\begin{tabular}{ll}
\hline Class & Compound \\
\hline
\end{tabular}

ab-PCM3-I-S1, ab-PCM3-I-S2, ab-PCM3-I-S3, ab-PCM3-I-S4, ab-PCM3-I-S5, ac-PCM2, ac-PCM3-I-S1, ac-PCM3-I-S2, ac-PCM3-I-S3, ac-PCM3-I-S4, ac-PCM3-I-S5, arabinose, carboxymethylated P. cocos polysaccharides, carboxymethylpachymaram, CMP, CS-PCS3-II, fucose, galactose, galacturonic acid,

Polysaccharides glucose, glucuronic acid, mannose, oxidized P. cocos polysaccharides, pachyman, PC-II, PCP3-I, PCP3-II, PCP4-I, PCP4-II, PCP-E, PCP-H, PCP-M, PCPS, PCP-U, PCS1, PCS2, PCS3-I, PCS3-II, PCS4-I, PCS4-II, PCSC, PCWPS, PCWPW, Pi-PCM, Pi-PCM0, Pi-PCM1, Pi-PCM2, Pi-PCM3-I, poria cocos polysaccharide-1(PCP-1), PPSW-1, rhamnose, rib, ribose, sum, wc-PCM0, wc-PCM1, wc-PCM2, xylose

$11 \beta$-ethoxydaedaleanic acid A, $15 \alpha$-hydroxy-3-oxolanosta-8,24-dien-21-oic acid, $15 \alpha$-hydroxydehydrotrametenolic acid, $15 \alpha$-hydroxydehydrotumulosic acid, $15 \alpha$-hydroxyeburiconic acid, 16-deoxyporicoic acid B, 16-hydroxy-3,24-dioxolanosta-7,9-dien-21-oic acid, 16-o-acetyl pachymic acid, $16 \alpha, 25$-dihydroxy dehydroeburiconic acid, 16a,25-dihydroxyeburiconic acid,

$16 \alpha, 27$-dihydroxydehydrotrametenoic acid, 16a,29-dihydroxy eburiconic acid, $16 \alpha$-acetoxy-26,27-dimethoxyl-lanosta-8,24-dien-21-oic acid, $16 \alpha$-acetoxy-lanosta-7,9,24-dien-21-oic acid, $16 \alpha$-acetoxy-lanosta-8,24-dien-21-oic acid, $16 \alpha$-acetoxypolyporenic acid C,

$16 \alpha$-acetyloxy-24-methylene-3-oxolanosta-7,9-dien-21-oic acid, $16 \alpha$-acetyloxye buriconic acid, $16 \alpha$-hydroxy-3-oxolanosta-8,24-dien-21-oic acid, 16 $\alpha$-hydroxydehydrotrametenonic aicd, $16 \alpha$-hydroxy eburiconic acid, 16 $\alpha$-hydroxy-lanosta-7,9,24-trien-21-oic acid, 16 $\alpha$-hydroxy-lanosta-8,24-dien-21-oic acid, $16 \alpha$-hydroxy trametenolic acid, 16,29-dihydroxy-3,4-seco-lanosta-4(28),8,24(31)-trien-3,21-dioic acid, 2'-3'-dihydrosorbicillin, 25,26-dihydroxy dehydropachymic acid, 25-hydroxy-3-epidehydrotumulosic acid, 25-hydroxy-3-epitumulosic acid, 25-hydroxy pachymic acid, 25-hydroxy polyporenic acid C, 25-hydroxyporicoic acid C, 25-hydroxyporicoic acid H, 25-methoxy-29-hydroxy poricoic acid HM, 25-methoxy poricoic acid A, 25 $\alpha$-hydroxytumulosic acid, 26-hydroxyporicoic acid DM, 26-hydroxy poricoic acid G, 29-hydroxydehydropachymic acid, 29-hydroxydehydrotumulosic acid, 3-(2-hydroxy acetoxy)-5 $\alpha, 8 \alpha$-peroxydehydrotumulosic acid, 3,4-seco-lanosta-4,7,9,24-tetraen-3,21-dioic acid, 31-hydyoxyl-16-o-acetylpachymic acid, 3-acetyloxy-16 $\alpha$-hydroxy trametenolic acid, 3-epi-(3'-hydroxy-3'-methylglutaryloxyl)-dehydrotumulosic acid, 3-epi-dehydropachymic acid, 3-epi-dehydrotumulosic acid, 3-epi-pachymic acid, 3-o-acetyl-16 $\alpha$,26-dihydroxytrametenolic acid, 3-o-acetyl-16 $\alpha$-hydroxydehydrotrametenolic acid, 3-o-acetyl-16 $\alpha$-hydroxytrametenolic acid, 3-o-acetyl-dehydroeburicoic acid, 3-oxo-16 $\alpha$-hydroxy-lanosta-7,9(11),24-trien-21-oic acid, 3-oxo-6,16 $\alpha$-dihydroxy-lanosta-7,9(11),24(31)-trien-21-oic acid, 3-oxo-lanosta-7,9(11),24(31)-trien-21-oic acid, 3-oxo-lanosta-7,9(11),24-trien-21-oic acid, $3 \alpha, 16 \alpha, 25$-Trihydroxylanosta-8,24-dien-21-oic acid, $3 \beta, 15 \alpha$-Bis(acetyloxy)-24-methylenelanost-8-en-21-oic acid, 3 $\beta, 15 \alpha$-dihydroxy-24-oxolanosta-8-en-21-oic acid, $3 \beta, 15 \alpha$-dihydroxy lanosta-7,9(11),24-triene-21-oic acid, $3 \beta, 16 \alpha$-bis(acetyloxy)-29-hydroxylanosta-8,24-dien-21-oic acid, $3 \beta, 16 \alpha$-dihydroxy-24-hydroxy methyl lanosta-7,9-dien-21-oic acid, 3 $\beta, 16 \alpha$-dihydroxy lanosta-7,9(11),24(31)-trien-21-oic acid, $3 \beta, 16 \alpha$-dihydroxylanosta-7,9(11),24-trien-21-oic acid, 3 $\beta, 26$-dihydroxy-lanosta-7,9,24-trien-21-oic acid, $3 \beta$-acetoxy-16 $\alpha, 26$-dihydroxylanosta-8,24-dien-21-oic acid, $3 \beta$-acetoxy-lanosta-7,9(11),24-trien-21-oic acid, $3 \beta$-acetyloxy-16 $\alpha$-hydroxy-24-oxolanost-7,9(11)-dien-21-oic acid,

$3 \beta$-acetyloxy-16 $\alpha$-hydroxy-24-oxolanost-8-en-21-oic acid,

$3 \beta$-hydroxy-16 $\alpha$-acetoxylanosta-7,9(11),24-trien-21-oic acid, 3 $\beta$-p-hydroxybenzoyldehydrotumulosic acid, $3 \alpha$-hydroxyl-26-methoxyl-8,24(31)-diene-21-oic acid, $3 \beta$-acetoxy-16 $\alpha$-hydroxy-lanosta-8,24-diene-21-oic acid, $5 \alpha, 8 \alpha$-Peroxydehydrotumulosic acid, 6,16 $\alpha$-dihydroxydehydroeburiconic acid,

6,16 $\alpha$-dihydroxydehydrotrametenonic acid, 6-hydroxy polyporenic acid C, $6 \alpha$-hydroxydehydropachymic acid, $6 \alpha$-hydroxy-polyporenic acid C, 9,11-dehydroergosterol peroxide,

6-hydroxy-3 $\beta, 16 \alpha$-diacetoxy-lanosta-7,9,24-tetraen-21-oic acid, acetoxyeburicoic acid, acetyl-eburicoic acid, ceanphytamic acid A, ceanphytamic acid B, daedaleanic acid A, daedaleanic acid B, dehydroeburic acid, dehydroeburicoic acid, dehydroeburicoic acid monoacetate, dehydroeburiconic acid, dehydropachymic acid, dehydrosulphurenic acid, dehydrotrametenolic acid, dehydrotrametenonic acid, dehydrotumulosic acid, dehypachymic acid, deyhdrotarmetenolic acid, eburicoic acid, eburicoic acid acetate, ergosterol 
peroxide, harzianone, hederagenin, hispindic acid B, lanosta-7,9,24-trien-21-oic acid, lanosta-8,24-dien-21-oic acid, me trametenolate, oleanic acid, pachymic acid, pinicolic acid, pinicolic acid $\mathrm{A}$, pinicolic acid $\mathrm{E}$, polyporenic acid $\mathrm{C}$, poriacosone $\mathrm{A}$, poriacosone $\mathrm{B}$, poricoic acid $\mathrm{A}$, poricoic acid $\mathrm{AE}$, poricoic acid AM, poricoic acid B, poricoic acid C, poricoic acid $\mathrm{CE}$, poricoic acid CM, poricoic acid $\mathrm{D}$, poricoic acid DM, poricoic acid E, poricoic acid F, poricoic acid G, poricoic acid GE, poricoic acid GM, poricoic acid h, poricoic acid $\mathrm{HE}$, poricoic acid $\mathrm{HM}$, poricoic $\mathrm{B}$, pregna-7-en-3 $\alpha, 11 \alpha, 15 \alpha, 20$-quadriol, trametenolic acid, tumulosic acid, ursolic acid

Alkaloids adenosine, thymine

(3S,6S)-3-[(1R)-1-hydroxyethyl]-6-(phenylmethyl)-2,5-piperazinedione, (5-formylfuran-2-yl) methyl

Others 2-(4-hydroxyphenyl) acetate, (5-formylfuran-2-yl) methyl 2-hydroxy propanoate, 5-hydroxy methylfurfural, sohiracillinone, sohirnone A, sorbicillin

Many methods have been developed for the analysis and the quality control of Licorice. The 58 phenolic acids including 11 new compounds (glycybridins A-K) were isolated from Licorice using nuclear magnetic resonance (NMR) and MS analyses combination with experimental and computed electronic circular dichroism data [97]. The 8 triterpenoids including glyuralsaponin A-G were separated from Licorice root by using ultraviolet (UV), infrared (IR), NMR spectrum and other technologies [98]. In addition, 122 chemical ingredients including glycyuralin A-F from the roots and stems of Licorice were isolated and identified adopting a combined strategy using NMR and MS spectroscopic data measurement [99].

We totally collected 260 components of Licorice [97]-[109], which were classified into 6 categories, including flavonoids (112 compounds, 43\%), triterpenoids (51 compounds, 20\%), phenolic acids (72 compounds, 28\%), coumarins (11 compounds, $4 \%$ ), stilbenoids (5 compounds, $2 \%$ ) and others (9 compounds, $3 \%$ ) (Figure 1(e), Table 5).

\section{The Chemical Constituents in Suanzaoren Decoction}

We used "Suanzaoren decoction", "Suan-Zao-Ren decoction", "Suan-ZaoRen-Tang”, “Zizyphus Combination”, "Suanzaoren Formulae”, "Suan Zao Ren Tang", "Ziziphus spinose decoction", "Suanzaoren prescription" as key words to search chemical components in SZRD, and classified them according to their chemical structure. Results showed that only five literatures on the ingredients of SZRD were reported. For example, 101 constituents are identified in Suan-Zao-Ren granule using UHPLC-Q-TOF-MS coupled with multiple data processing approaches [110]. These 48 components were characterized in SZRD using liquid chromatography time-of-flight mass spectrometry (LC-Q/TOFMS) and liquid chromatography coupled with ion trap mass spectrometry (LC-IT-MS) technology and 31 compounds were identified for the first time [111]. The 22 chemical compounds were isolated in SZRD by using ultra-performance liquid chromatography coupled with electrospray ionization/quadrupole-time-of-flight mass spectrometry (UPLC-ESI-Q-TOF-MS) 
Table 5. Chemical constituents in Gancao (Licorice, Glycyrrhiza uralensis Fisch., Glycyrrhiza inflata Bat. or Glycyrrhiza glabra L.).

\begin{tabular}{|c|c|}
\hline Class & Compound \\
\hline Flavonoids & 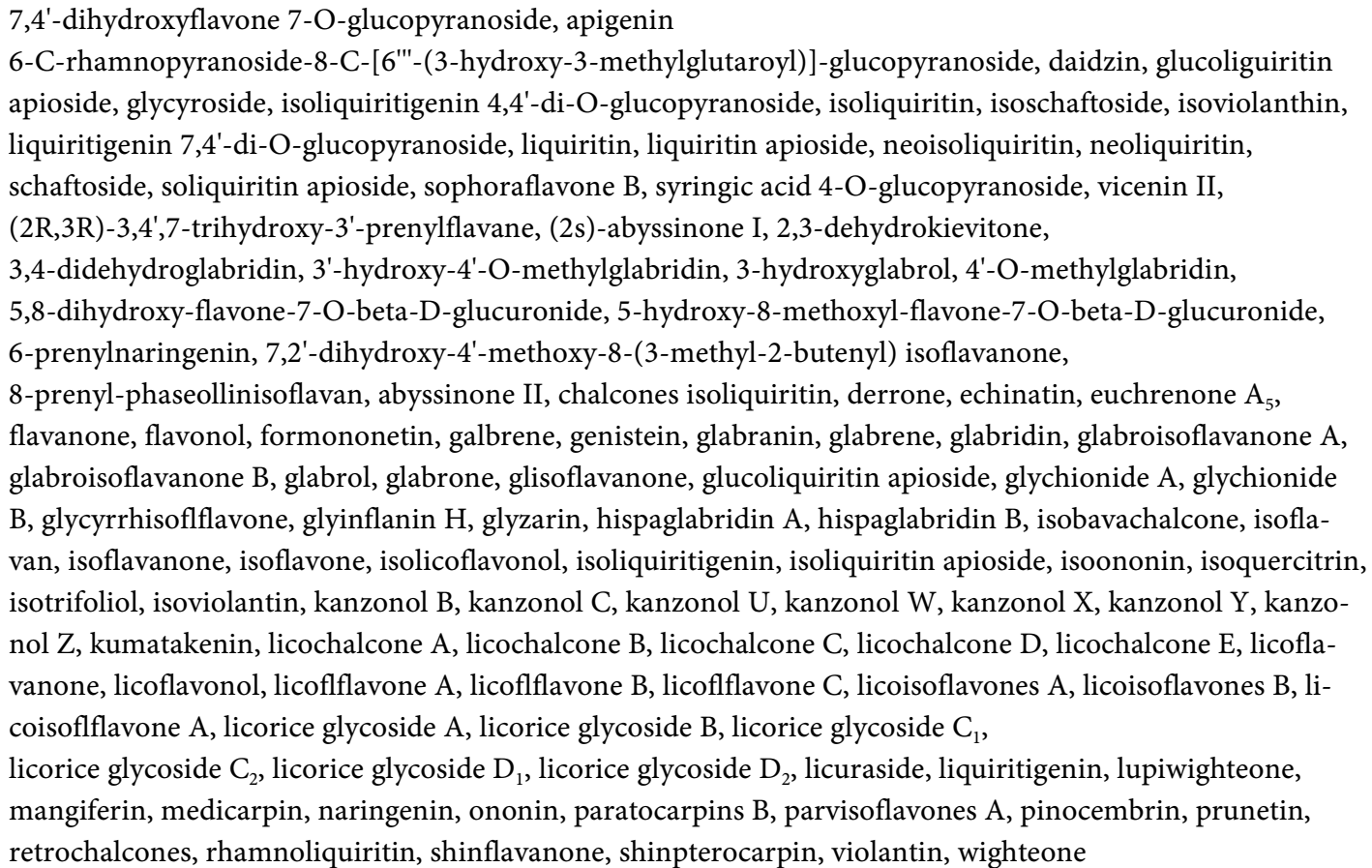 \\
\hline
\end{tabular}

12-acetoxyganoderic acid F, $18 \beta$-glycyrrhetinic acid, $22 \beta$-acetoxylglycyrrhizin, 24-hydroxy-licorice saponin A3, 3 $\beta$-O-[ $\beta$-D-glucuronopyranosyl- $(1 \rightarrow 2)-\beta$-D-glucuronopyranosyl]-24-hydroxylglabrolide, glabrolide, glycyrrhetic acid, glycyrrhetic acid-3-O-mono- $\beta$-d-glucuronide, glycyrrhizic acid, glyuralsaponin $\mathrm{A}$, glyuralsaponin B, glyuralsaponin C, glyuralsaponin D, glyuralsaponin E, glyuralsaponin F, glyuralsaponin G, glyuralsaponin $\mathrm{H}$, isoglycyrrhizin, licorice saponin $\mathrm{A}_{3}$, licorice saponin $\mathrm{B}_{2}$, licorice saponin $\mathrm{C}_{2}$, licorice saponin $E$, licorice saponin $E_{2}$, licorice saponin $G_{2}$, licorice saponin $\mathrm{N}_{4}$, squasapogenol $G P-B_{2}$, uralsaponin $\mathrm{B}$, uralsaponin $\mathrm{F}$, yunganoside $\mathrm{K}_{2}, 22 \beta$-acetoxyglycyrrhizin, $22 \beta$-acetoxyl-glycyrrhaldehyde, 3-O-glucuronopyranosyl-glycyrrhetinic acid,

$3 \beta$-O-[glucuronopyranosyl-(1 $\rightarrow 2)$-glucuronopyranosyl]-olean-9,12-diene-30-oic-acid, araboglycyrrhizin, glycyrrhetinic acid, glycyrrhizin, licorice-saponin $\mathrm{H}_{2}$, licorice-saponin $\mathrm{J}_{2}$, uralsaponin $\mathrm{C}$, uralsaponin $\mathrm{M}$, uralsaponin $\mathrm{N}$, uralsaponin $\mathrm{P}$, uralsaponin $\mathrm{Q}$, uralsaponin $\mathrm{R}$, uralsaponin $\mathrm{S}$, uralsaponin $\mathrm{T}$, uralsaponin $\mathrm{U}$, uralsaponin $\mathrm{V}$, uralsaponin $\mathrm{W}$, uralsaponin $\mathrm{X}$, uralsaponin $\mathrm{Y}$

11b-hydroxy-11b,1-dihydromedicarpin, 1-methoxyphaseollin, 2'-hydroxyisolupalbigenin, 2-one-4-methoxy-pyran, 3-methoxy-9-hydroxy-pterocarpan,

5,7,4'-trihydroxy-3'(3-methylbut-2-enyl)-3-methoxy flavone, 6,8-diprenylgenistein, 6-C-prenylorobol, 7-methoxy-2',4'-dihydroxy isoflavone, 7-O-methylluteone, abiochanin A, allolicoisoflavone $\mathrm{B}$, angustone A, daidzein, dehydroglyasperin C, dehydroglyasperin D, gancaonin I, gancaonin L, genkwanin, glicophenone, glicoricone, glyasperin C, glyasperin D, glycybridins A, glycybridins B, glycybridins C, glycybridins D,

Phenolic acids glycybridins E, glycybridins F, glycybridins G, glycybridins H, glycybridins I, glycybridins J, glycybridins K, glycyfuranocoumarin A, glycyfuranocoumarin B, glycyfuranocoumarin C, glycyfuranocoumarone A, glycyrol, glycyrrhiza-isoflavone C, glycyuralin A, glycyuralin B, glycyuralin C, glycyuralin D, glycyuralin E, glycyuralin F, glyurallin A, hirtellanine I, homobutein, isoangustone A, isoderrone, isoglabrone, isoglycycoumarin, isoglycyrol, isolupalbigenin, kaempferol, kaempferol 3-O-methyl ether, kumatakenin B, licoarylcoumarin, licocoumarone, licoflavone A, licoisoflavanone, licoisoflavone A, licoisoflavone B, licoricidin, licoricone, luteone, phaseollin, pratensein, semilicoisoflavone B, topazolin, uralenol 


\section{Continued}

\begin{tabular}{|c|c|}
\hline Stilbenoids & $\begin{array}{l}\text { dihydro-3,3',4'-trihydroxy-5-O-isopentenyl-6-isopentenylstilbene, } \\
\text { dihydro-3,3'-dihydroxy-5 } \beta \text {-d-O-glucopyranosyloxy-4'-methoxystilbene, } \\
\text { dihydro-3,5,3'-trihydroxy-4'-methoxystilbene, dihydro-3,5-dihydroxy-4'-acetoxy-5'-isopentenylstilbene, } \\
\text { dihydrostilbenes }\end{array}$ \\
\hline Coumarins & $\begin{array}{l}\text { glabrocoumarin, glabrocoumarone A, glabrocoumarone B, glycocoumarin, glycycoumarin, glycyrin, } \\
\text { herniarin, licofuranocoumarin, licopyranocoumarin, liqcoumarin, umbelliferone }\end{array}$ \\
\hline Others & $\begin{array}{l}\text { [6",6"-dimethyl pyrano }(2 ", 3 ": 4,5)]-3 \text { '- } \gamma, \gamma \text {-dimethylallyl-2',3,4'-trihydroxychalcone, } \\
1 ", 2 \text {-dehydrocyclokievitone, } 2 \text { '-O-demethybidwillol, dehydroglyceollin I, erybacin, } \\
\text { licoagrocarpin, licoagrochalcone A, xambioona, gup-II }\end{array}$ \\
\hline
\end{tabular}

method with MassLynx ${ }^{\mathrm{TM}}$ MassFragment [112]. There were 13 active marker compounds (neomangiferin, mangiferin, spinosin, liquiritin apioside, liquiritin, 6"'-feruloylspinosin, senkyunolide I, timosaponin BII, isoliquiritoside, timosaponin $\mathrm{C}$, jujuboside $\mathrm{A}$, jujuboside $\mathrm{B}$, and timosaponin $\mathrm{AIII}$ ) were separated in diverse SZRD including lab-prepared Suanzaoren oral liquid, Suanzaoren mixture, and clinical Suanzaoren granules using high-performance liquid chromatography with diode array detection and evaporative light scattering detection (HPLC-DAD-ELSD) [113]. In addition, 11 chemical components (neomangiferin, mangiferin, spinosin, liquiritin apioside, liquiritin, fumalic acid, 6"'-feruloylspinosin, senkyunolide I, isoliquiritin, glycyrrhizic acid and senkyunolide A) of SZRD in different batches, including SZRD extracts, lab-made SZRD granules and clinical medicine, were determined by HPLC-PDA, which indicated that HPLC-PDA method would be helpful to improve quality evaluation and quality control in productive processes [114]. The 8 compounds (jujuboside, spinosin, ferulic acid, senkyunolide I, sarsasapogenin, mangiferin, liquiritoside and glycyrrhizic acid) were considered chemical quality control standard of SZRD [115].

We totally collected 145 compounds of SZRD in all [110] [111] [112] [113] [114], which were divided into 10 categories, including flavonoids (53 compounds, $36 \%$ ), alkaloids ( 2 compounds, $1 \%)$, coumarins (7 compounds, $5 \%$ ), phenolic acids (4 compounds, $3 \%$ ), phthalide (14 compounds, 10\%), triterpenoids (33 compounds, 23\%), steroids (19 compounds, 13\%), volatile oils (7 compounds, $5 \%$ ), polysaccharide (1 compound, $1 \%$ ) and others (5 compounds, $3 \%$ ) (Figure 1(f), Table 6). Flavonoids, one of the main active ingredients found at present, contained the largest number of ingredients in SZRD [116].

\section{Comparative Analysis of Chemical Constituents in Herbs and Suanzaoren Decoction}

\subsection{Comparative Analysis of Chemical Constituents in Herbs}

We obtained a total of 1104 chemical components without duplication in 5 herbs (Figure 2(a)). Adenosine existed in Ziziphi Spinosae Semen, Chuanxiong Rhizoma, Poria and Anemarrhenae rhizoma. Oleic acid existed in Anemarrhenae 
Table 6. Chemical constituents in suanzaoren decoction.

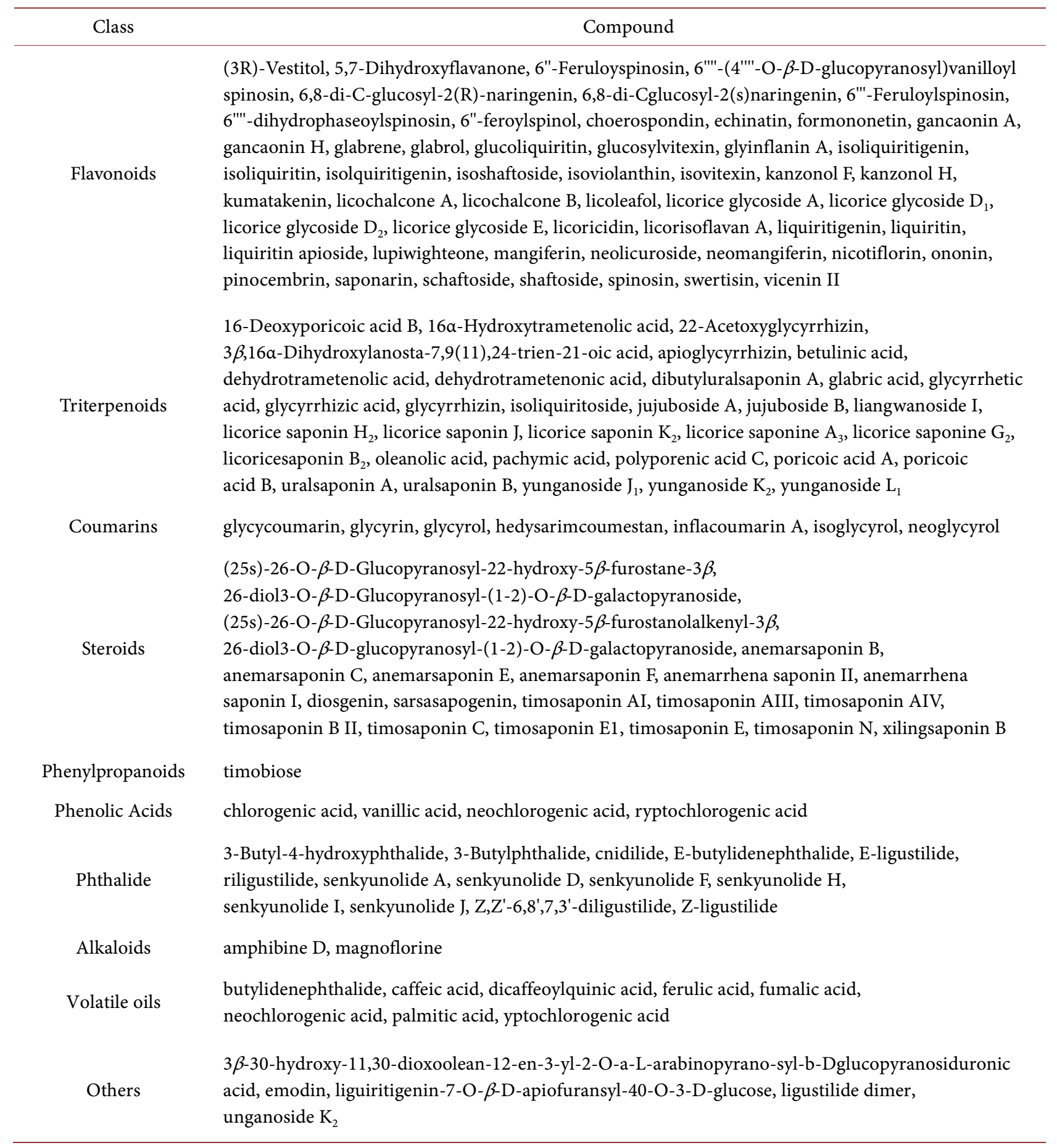

rhizoma, Chuanxiong Rhizoma and Ziziphi Spinosae Semen.

There are 8 common components presenting in Chuanxiong Rhizoma and Poria, which are galacturonic acid, mannose, arabinose, ergosterol peroxide, rhamnose, galactose, glucuronic acid, and glucose. Ziziphi Spinosae Semen and Chuanxiong Rhizoma contain linoleic acid and protocatechuic acid. Ziziphi Spinosae Semen and Anemarrhenae rhizoma contain tryptophan and isovitexin. 


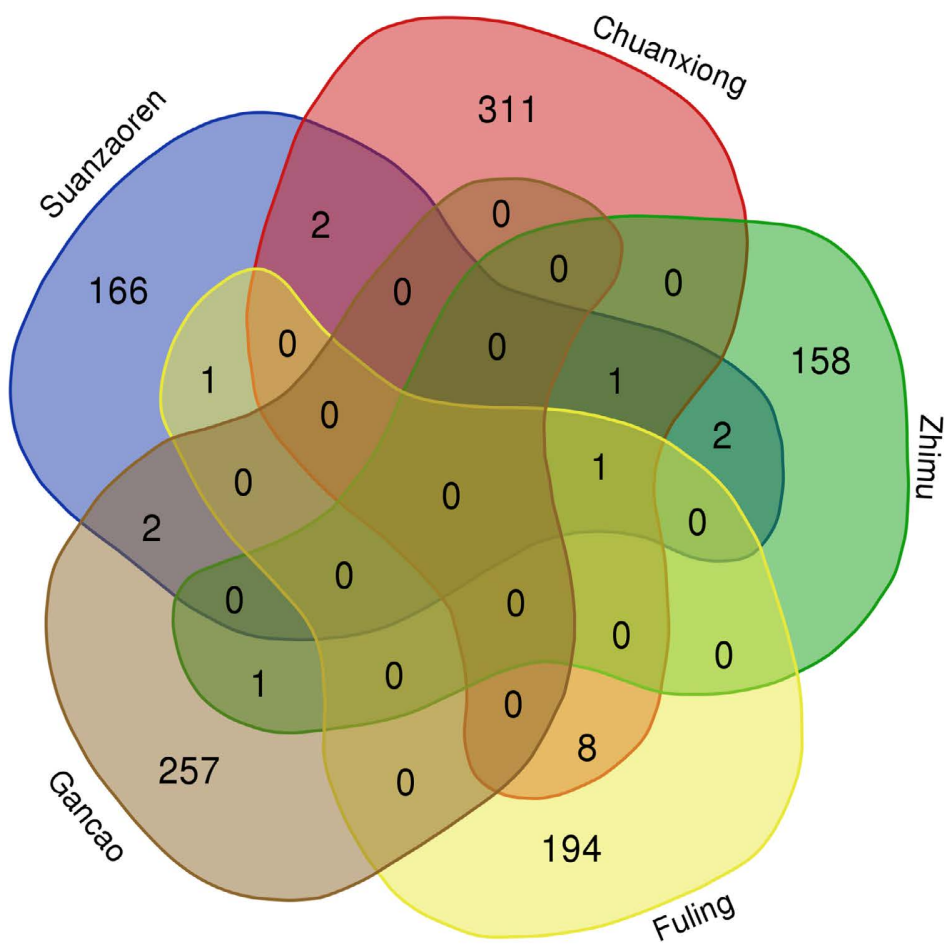

(a)

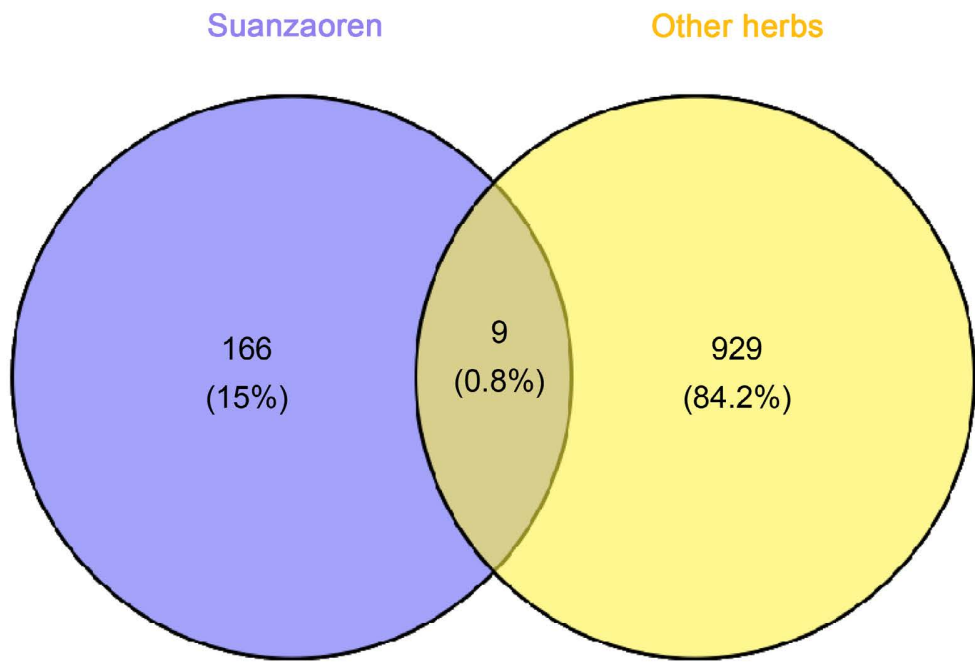

(b)

Figure 2. Chemical compounds in five-single herbs of Suanzaoren decoction. (a) Intersection of compounds in five-single herbs; (b) Intersection of compounds between Ziziphi Spinosae Semen and other four herbs.

Ziziphi Spinosae Semen and Licorice include isoquercitrin and vicenin II. Ziziphi Spinosae Semen and Poria contain ursolic acid. Anemarrhenae rhizoma and Licorice contain only one component mangiferin (Figure 2(a)).

In SZRD, Ziziphi Spinosae Semen seed is "Jun" (the monarch). There are 175 chemical compounds in Ziziphi Spinosae Semen seed, accounting for $15.8 \%$ in 
all 5 herbs (Figure 2(b)). When compared with total compounds in the other four herbs, there are 9 common compounds with $0.8 \%$ of total compounds in all 5 herbs, including adenosine, tryptophan, isoquercitrin, isovitexin, vicenin II, linoleic acid, oleic acid, protocatechuic acid and ursolic acid (Figure 2(b)).

\subsection{Comparative Analysis of Chemical Constituents between Suanzaoren Decoction and Herbs}

\subsubsection{Ingredients between Suanzaoren Decoction and Herbs}

After comparing 145 compounds in SZRD with 1104 in 5 herbs, We found there were 80 common components which accounted for $6.8 \%$ of total compounds in all herbs and $55.2 \%$ of compounds in SZRD (Figure 3(a)), containing 7 categories, including 27 flavonoids, 17 triterpenoids, 12 steroids, 5 volatile oils, 4 coumarins, 14 phenolic acids and 1 alkaloid (Figure 3(b)) and found that 14 compounds came from Ziziphi Spinosae Semen, 18 from Chuanxiong Rhizoma, 15 from Anemarrhenae rhizoma, 9 from Poria and 27 from Licorice.

In addition, 65 compounds in SZRD are not contained in Ziziphi Spinosae Semen, Anemarrhenae rhizoma, Chuanxiong Rhizoma, Poria and Licorice (Figure 3(c)). These distinctive compounds account for 5.6\% of total compounds in all herbs and $44.8 \%$ of SZRD compounds (Figure 3(a)), and include alkaloids ( 1 compound), coumarins ( 3 compounds), flavonoids ( 25 compounds), phenolic acids (1 compound), phthalides (2 compounds), polysaccharides (1 compound), steroids (7 compounds), triterpenoids (17 compounds), volatile oils ( 3 compounds) and others ( 5 compounds). The number of flavonoids is the highest among SZRD, followed by triterpenoids, suggesting ingredients of SZRD are not simply the sum of the single ingredients and new compounds will produce after decocting SZRD.

The amounts of volatile oils, alkaloids and polysaccharides are decreased while the number of flavonoids is increased in SZRD compared with 5 single herbs (Figure 4(a) and Figure 4(b)). In general, there are many water-soluble compounds in SZRD, such as flavonoids, triterpenoids, accounting for $59.31 \%$. However, triterpenoids, flavonoids and volatile oils account for $22.95 \%, 18.24 \%$ and $15.93 \%$ respectively in 5 single herbs. Amino acids, ceramides and cerebrosides, lignans, nucleotides, steroids and stilbenides are not found in SZRD. We inferred it might due to the extraction of SZRD just exists in aqueous decoction, the proportion of components with larger polarity is significantly increased, while the proportion of components with weak polarity is relatively low. On the other hand, it may because the lack of literature on the components of SZRD, and the literatures are just for the analysis of specific components and lack researches on specific components.

\subsubsection{Ingredients between Suanzaoren Decoction and Ziziphi Spinosae Semen}

We compared 145 compounds contained in SZRD with 175 in Ziziphi Spinosae Semen, the monarch of SZRD (Figure $4(c)$ ). There are 14 common components, 


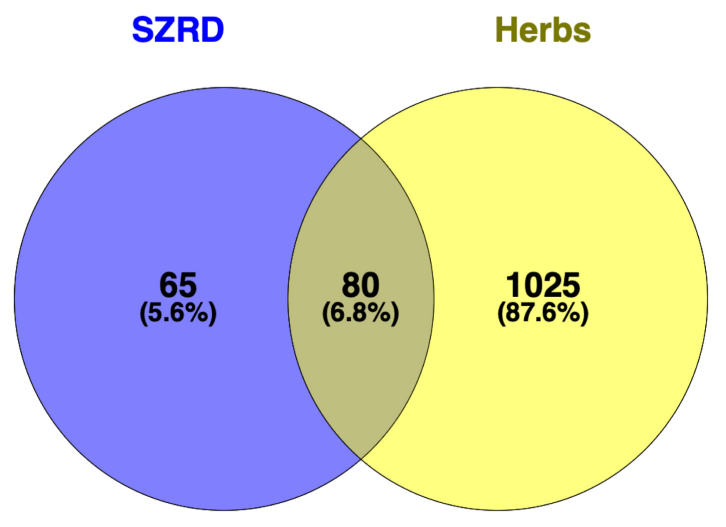

(a)
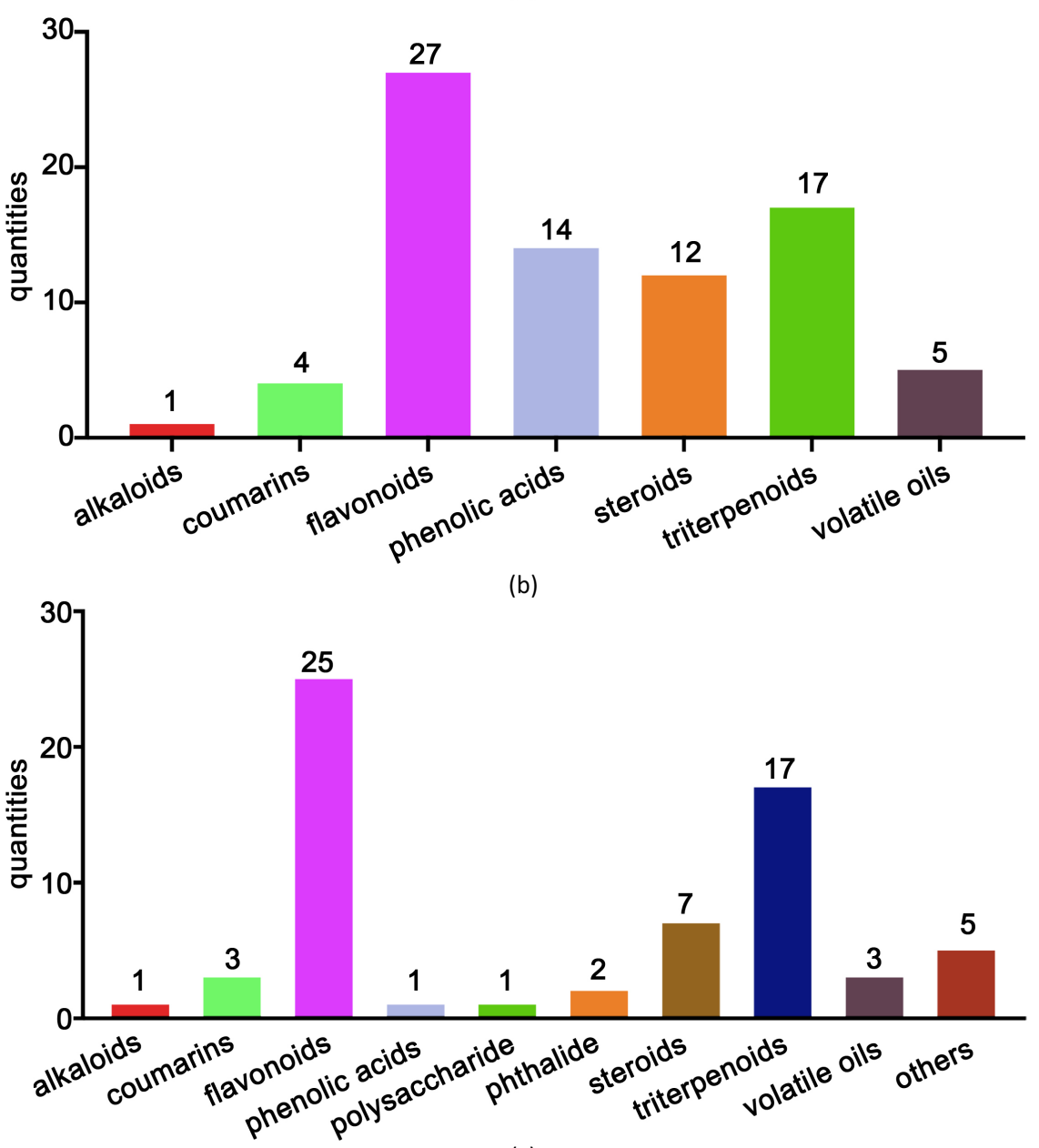

(c)

Figure 3. Chemical compounds in suanzaoren decoction and its herbs. (a) Intersection of compounds in SZRD and its herbs; (b) Classification of same compounds of SZRD and its herbs; (c) Classification of different compounds of SZRD and its herbs.

accounting for 9.7\% in SZRD, which are flavonoids (6"'-Feruloylspinosin, glucosylvitexin, isovitexin, nicotiflorin, saponarin, spinosin, wertisin, vicenin II), triterpenoids (betulinic acid, jujuboside A, jujuboside B, oleanolic acid), alkaloid (magnoflorine) and volatile oil (palmitic acid), while it has 131 different 


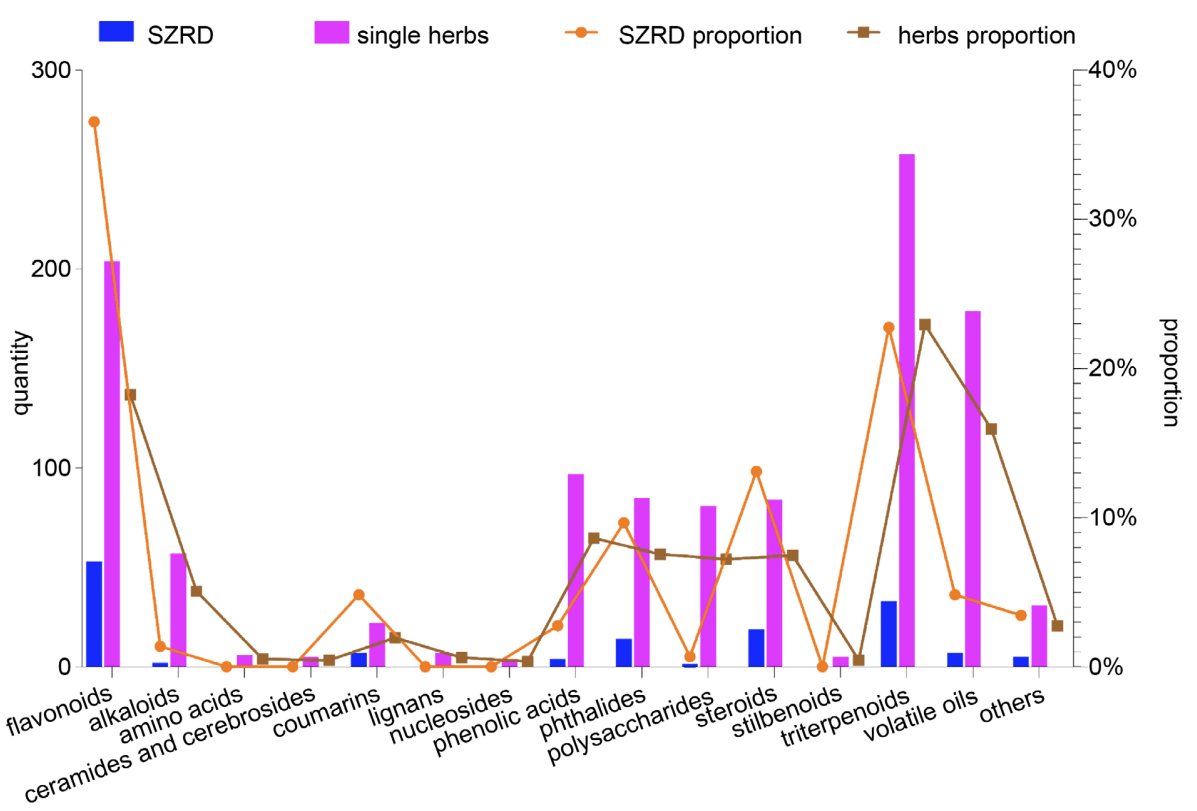

(a)

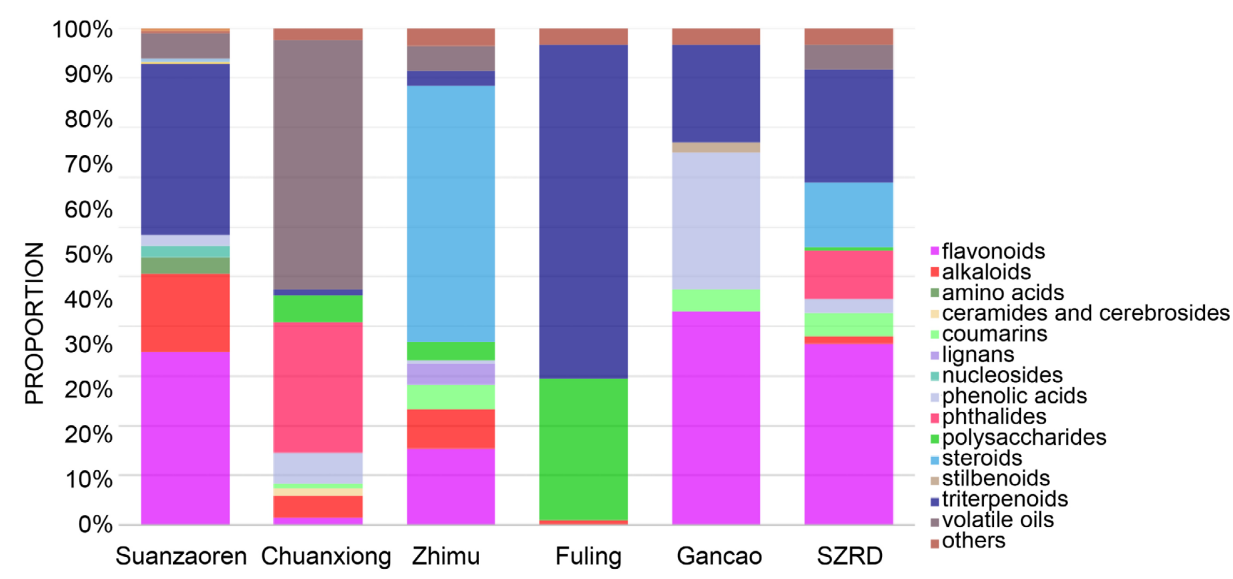

(b)

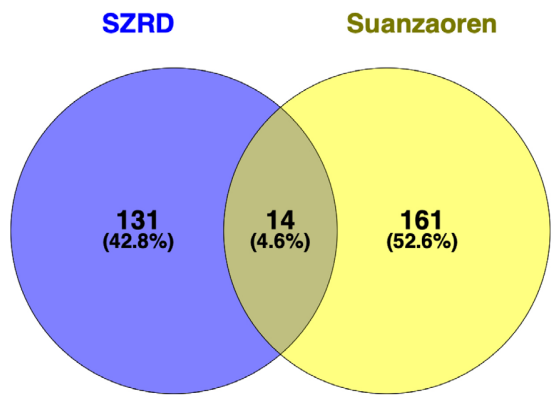

(c)

Figure 4. Chemical compounds in suanzaoren decoction and five-single herbs. (a) Proportion and quantity of various ingredients in total ingredients of herbs and SZRD. The column chart shows the quantity of various ingredients in the total compounds of herbs or the prescription, while line chart shows the proportion of various ingredients in the total compounds of herbs or the prescription. The left ordinate indicates quantity and the right ordinate indicates proportion. (b) Proportion of various ingredients in herbs and SZRD. (c) Intersection compounds of SZRD and Ziziphi Spinosae Semen. 
compounds (90.3\%) (Figure 4(c)), including flavonoids (45 compounds), alkaloid (1 compound), coumarins (7 compounds), phenolic acids (4 compounds), phthalides (14 compounds), triterpenoids (29 compounds), steroids (19 compounds), volatile oils ( 6 compounds), polysaccharide ( 1 compound) and others (5 compounds) (Figure 4(a) and Figure 4(b)).

\section{Summary and Perspectives}

In SZRD, Ziziphi Spinosae Semen is the monarch drug, Chuanxiong Rhizoma and Poria are minister drugs, Anemarrhenae rhizoma is an adjuvant, Licorice is conductant drug, which can reconcil various drugs. Totally, we collected 145 and 1104 chemical ingredients in SZRD and its five-single herbs respectively and found 80 common ingredients between SZRD and five-single herbs, while 65 different compounds only in SZRD. Taken together, our study elucidated that components of SZRD were not simply the sum of one in every single herb. Based on the analysis of the relationship of ingredients between five-single herbs and the whole prescription, we found that the problems existed in the chemical research of SZRD and possible further research direction.

\subsection{What Has Already Been Shown to Be Not Promising}

1) As the chemical profile of the decoction is dependent on the quality of the plant materials, each compound probably was a wide range of concentrations. Therefore, we highlighted names, numbers and variabilities of compounds in the five herbs and SZRD, nothing is said about the quantities or actual concentrations of these compounds in this review.

2) As different methods have been used, it will be difficult to compare the results of the various studies. There was an effect of the experimental conditions on the overall extracted chemical profile of the decoction. It may be different conditions and time of soaking and decocting, different extraction solvents, different apparatus and parameters to identify SZRD and five-single herbs. That means this number of compounds is very much based on the sensitivity and selectivity of the methods of analysis. For example, the compounds mentioned represent a wide range of polarities, and many of them from five-single herbs will probably not being extraction extracted in SZRD.

3) The variability in the raw materials had an effect on the plant materials on the overall extracted chemical profile of the decoction. For example, the different local growing environments of plants.

4) The different understanding of researchers on the compounds, and global chemical components had an effect on compound being obtained and identified in herbs.

5) It is unbalanced at present of the studies among Ziziphi Spinosae Semen, Chuanxiong Rhizoma, Anemarrhenae rhizoma, Poria, Licorice and SZRD, that mean there were more compounds in herbs with many reports than that with few reports. It was lack of global chemical components for each herb and SZRD. 


\subsection{What Looks Promising}

1) As SZRD is composed of Ziziphi Spinosae Semen, Chuanxiong Rhizoma, Anemarrhenae rhizoma, Poria, Licorice, it is necessary to perform parallel studies among SZRD and its herbs.

2) As pharmacological activity has a dose-response curve, moreover synergy and antagonistic effect may occur between compounds in certain ratios, it is the biological activity of the individual compounds and their concentration that determine the activity of the final preparation. Therefore, it is necessary to study phytochemistry and pharmacological activities at the same time.

3) What is needed is to integrate the data on activity of extracts and pure compounds with the data on traditional use and the data on the chemistry.

\subsection{The Limitations of This Article}

1) Compounds collection of SZRD and five-single herbs were mainly based on the literature in the past five years, but few had been reported more than five years.

2) This paper did not carry out a classification analysis for non-English name compounds.

3) For the chemical composition of SZRD and its five-single herbs, we just simply classified them based on their chemical structures while not carrying out subcategory analysis.

Although these results are exploratory and need to be interpreted with caution, our study provides important information that may help offer subsequent studies to determine the compounds and explore pharmacology research about SZRD. This review may provide the reference for phytochemistry and pharmacology researches of SZRD, and also point out the direction for its further research.

\section{Acknowledgements}

This work was supported by the National Natural Science Foundation of China (No. 81974555).

\section{Consent for Publication}

All authors have given consent for publication.

\section{Competing interests}

The authors declare that they have no competing interests.

\section{References}

[1] Li, L. (2019) Clinical Observation on Suanzaoren Decoction in Treating Insomnia. Modern Medicine and Health Research, 3, 56-59.

[2] Singh, A. and Zhao, K. (2017) Treatment of Insomnia with Traditional Chinese Herbal Medicine. International Review of Neurobiology, 135, 97-115. 
https://doi.org/10.1016/bs.irn.2017.02.006

[3] Zhou, Q.H., Wang, H.L., Zhou, X.L., Xu, M.B., Zhang, H.F., Huang, L.B., Zheng, G.Q. and Lin, Y. (2017) Efficacy and Safety of Suanzaoren Decoction for Chronic Insomnia Disorder in Adults: Study Protocol for Randomised, Double-Blind, DoubleDummy, Placebo-Controlled Trial. BMJ Open, 7, e014280.

https://doi.org/10.1136/bmjopen-2016-014280

[4] Hu, K. and Hu, X. (2019) Effect of Suanzaoren Decoction on Insomnia after Stroke. Contemporary Medical Symposium, 17, 200-201.

[5] Yang, T.Y., Tang, H., Qin, Y.Q. and Song, P.X. (2016) Effective Observation on Treating 26 Cases of Insomnia after Spinal Cord Injury with the Suanzaoren Decoction. Clinical Journal of Chinese Medicine, 8, 79-80.

[6] Yang, Y. (2016) Effect of Suanzaoren Decoction on Hyperthyroidism and Insomnia. Guide of China Medicine, 14, 213.

[7] Yeh, C.H., Arnold, C.K., Chen, Y.H. and Lai, J.N. (2011) Suan Zao Ren Tang as an Original Treatment for Sleep Difficulty in Climacteric Women: A Prospective Clinical Observation. Evidence-Based Complementary and Alternative Medicine, 2011, Article ID: 673813. https://doi.org/10.1155/2011/673813

[8] Li, J.Y. and Wang, X. (2004) Clinical Analysis of Suanzaoren Decoction in the Treatment of Generalized Anxiety Disorder. Journal of Shandong University of Traditional Chinese Medicine, 28, 438-439.

[9] Wang, Q. (2018) Effects of Addition and Subtraction Suanzaoren Decoction Treatment on Clinical Symptoms and Sleep Quality for Patients with Maintenance Hemodialysis Complicated with Depression. Journal of Sichuan of Traditional Chinese Medicine, 36, 104-106.

[10] Li, D. (2018) Clinical Observation on Suanzaoren Decoction for the Treatment of 48 Cases of Menopausal Syndrome. Journal of Clinical Medical, 5, 82-83.

[11] Sun, Z. and Zhang, X. (1998) Experience of Suanzaoren Decoction in Treating Intractable Premature Ventricular Contractions. Shandong Journal of Traditional Chinese Medicine, 17, 112-113.

[12] Sun, H. and Yang, L. (2000) Suanzaoren Decoction in Treating Congenital Non-Hemolytic Jaundice. Heilongiiang Journal of Traditional Chinese Medicine, 5, 36-37.

[13] Huang, D. and Hui, Z. (2017) SuanZaoRen Decoction Jointed with Estazolam in Treating 43 Patients with Insomnia of Liver Depression and Blood Deficiency Pattern. Western Journal of Traditional Chinese Medicine, 30, 93-95.

[14] Liu, L.Y. (2018) Study on the Effect of Suanzaoren Decoction Combined with Zhizizhi Decoction on Anxiety-Induced Insomnia. China Practical Medicine, 13, 138-139.

[15] Cai, C., Li, A., Zhang, R., Ma, C. and Feng, L. (2020) Effects of Suanzaoren Decoction and Sweet Dream Oral Liquid on Neuroendocrine, Cognitive Function and Deep Sleep in Patients with Sleep Disorder. Chinese Archives of Traditional Chinese Medicine, 38, 210-212. http://kns.cnki.net/kcms/detail/21.1546.R.20190814.1535.071.html

[16] Li, Y. and Yuan, F. (2019) Effect of Ziziphi Spinosae Decoction Combined with Acupuncture on Insomnia and Anxiety of Uremia Patients Undergoing Maintenance Dialysis. Journal of Liaoning University of TCM, 21, 133-136.

[17] Ji, S. (2019) Observation on the Effect of Suanzaoren Decoction Combined with Deanxit in the Treatment of Generalized Anxiety Disorder. Inner Mongolia Journal of Traditional Chinese Medicine, 38, 48-49. 
[18] Jang, X., Xu, H., Zhou, H. and Guo, Q. (2020) Suanzaoren Decoction in the Treatment of 30 Cases of Generalized Anxiety Disorder of Yin Deficiency and Fire Excitement. Journal of North Pharmacy, 17, 83-84.

[19] Zhou, Z. (2016) Suanzaoren Decoction Combined with Flupentixol and Melitracen Tablets and Oryzanol in the Treatment of Perimenopausal Depression Randomized Controlled Study. Journal of Practical Traditional Chinese Internal Medicine, 30, 55-57.

[20] Zhang, Y. (2017) Observation on the Curative Effect of Guipi Decoction Combined with Suanzaoren Decoction in the Treatment of Depression. Guangming Journal of Chinese Medicine, 32, 512-514.

[21] Liu, Y. (2017) Clinical Observation on 36 Cases of Perimenopausal Syndrome Treated with Danzhi Xiaoyao Powder and Suanzaoren Decoction. Hunan Journal of Traditional Chinese Medicine, 33, 58-60.

[22] Niu, X., He, B., Du, Y., Sui, Z., Rong, W., Wang, X., Li, Q. and Bi, K. (2018) The Investigation of Immunoprotective and Sedative Hypnotic Effect of Total Polysaccharide from Suanzaoren Decoction by Serum Metabonomics Approach. Journal of Chromatography B: Analytical Technologies in the Biomedical and Life Sciences, 1086, 29-37. https://doi.org/10.1016/j.jchromb.2018.04.004

[23] Wang, D., Li, Q., Liu, R., Xu, H., Yin, Y., Wang, Y., Wang, H. and Bi, K. (2019) Quality Control of Semen Ziziphi Spinosae Standard Decoction Based on Determination of Multi-Components Using TOF-MS/MS and UPLC-PDA Technology. Journal of Pharmaceutical Analysis, 9, 406-413. https://doi.org/10.1016/j.jpha.2019.01.001

[24] Zhang, F.X., Li, M. and Qiao, L.R. (2016) Rapid Characterization of Ziziphi Spinosae Semen by UPLC-Q-TOF-MS with Novel Informatics Platform and Its Application in Evaluation of Two Seeds from Ziziphus Species. Journal of Pharmaceutical \& Biomedical Analysis, 122, 59-80. https://doi.org/10.1016/j.jpba.2016.01.047

[25] Zhu, X., Liu, X., Pei, K., Duan, Y., Zhu, H., Ma, J., Xu, Y., Wu, Z., Zhou, Q. and Cai, B. (2018) Development of an Analytical Strategy to Identify and Classify the Global Chemical Constituents of Ziziphi Spinosae Semen by Using UHPLC with Quadrupole Time-of-Flight Mass Spectrometry Combined with Multiple Data-Processing Approaches. Journal of Separation Science, 41, 3389-3396.

https://doi.org/10.1002/jssc.201800171

[26] Zhao, J., Li, S.P., Yang, F.Q., Li, P. and Wang, Y.T. (2006) Simultaneous Determination of Saponins and Fatty Acids in Ziziphus jujuba (Suanzaoren) by High Performance Liquid Chromatography-Evaporative Light Scattering Detection and Pressurized Liquid Extraction. Journal of Chromatography A, 1108, 188-194.

https://doi.org/10.1016/j.chroma.2005.12.104

[27] Xie, Y.Y., Xu, Z.L., Wang, H., Kano, Y. and Yuan, D. (2011) A Novel Spinosin Derivative from Semen Ziziphi Spinosae. Journal of Asian Natural Products Research, 13, 1151-1157. https://doi.org/10.1080/10286020.2011.623128

[28] Xie, J., Zhang, Y., Wang, L., Qi, W. and Zhang, M. (2012) Composition of Fatty Oils from Semen Ziziphi Spinosae and Its Cardiotonic Effect on Isolated Toad Hearts. Natural Product Research, 26, 479-483. https://doi.org/10.1080/14786419.2010.516433

[29] Wang, Y., Ding, B., Luo, D., Chen, L.Y., Hou, Y.L., Dai, Y. and Yao, X.S. (2013) New Triterpene Glycosides from Ziziphi Spinosae Semen. Fitoterapia, 90, 185-191. https://doi.org/10.1016/j.fitote.2013.07.013

[30] Li, M., Wang, Y., Li, C., Dai, Y. and Yao, X.S. (2014) A New Flavone C-Glycoside 
from Ziziphi Spinosae Semen. Chinese Traditional and Herbal Drugs, 45, 2588-2592. (In Chinese)

[31] Wu, Y., Chen, M., Du, M.B., Yue, C.H., Li, Y.Y., Zhu, M., Liu, C., Wang, D.Y., Liu, J.G. and Hu, Y.L. (2014) Chemical Constituents from the Fruit of Ziziphus jujuba Mill. var. spinosa. Biochemical Systematics and Ecology, 57, 6-10. https://doi.org/10.1016/j.bse.2014.07.009

[32] Wang, Q. (2015) Research Progress on the Chemical Compositions and Application of Semen Ziziphi Spinosae. Heilongjiang Medicine Journal, 28, 259-261. (In Chinese)

[33] Geng, X. and Li, T.L. (2016) Progress on Chemical Composition and Pharmacological Activities of Semen Zizyphi Spinosae. Acta Chinese Medicine and Pharmacolo$g y, 44,84-86$. (In Chinese)

[34] Liu, S., Tang, Z., Cui, C., Liu, H., Liang, Y., Zhang, Y., Wang, M. and Gu, C. (2016) The Research Progress of Chemical Composition of SuanZaoRen. Western Journal of Traditional Chinese Medicine, 29, 143-146. (In Chinese)

[35] Liu, X., Zhu, X., Zhu, H., Xie, L., Ma, J., Xu, Y., Zhou, Q., Wu, Z. and Cai, B. (2018) Simultaneous Quantification of Six Bioactive Components in Decoction of Ziziphi spinosae Semen Using Ultrahigh Performance Liquid Chromatography Coupled with Triple-Quadrupole Mass Spectrometry. Journal of Analytical Methods in Chemistry, 2018, Article ID: 8397818. https://doi.org/10.1155/2018/8397818

[36] Mao, Y.N., Wu, W.Q., Kang, Y., Wang, F.F. and Liu, Y. (2018) Rapid Analysis of Chemical Components in Zizyphi Spinosae Semen by UHPLC-LTQ-Orbitrap-MS. China Journal of Chinese Materia Medica, 43, 4884-4891. (In Chinese)

[37] Du, C.H., Cui, X.F., Pei, X.P., Yan, Y. and Qin, X.M. (2019) Research Progress on Ziziphi Spinosae Semen Saponins and Its Biological Action on Nervous System. Chinese Traditional and Herbal Drugs, 50, 1258-1268. (In Chinese)

[38] Zhou, S.N., Chen, A.J., Guo, B.L., Zhang, Q.Y. and Qin, X.L. (2019) Determination of Two Flavonoids and Two Saponins in Ziziphi Spinosae Semen from Different Producing Areas. Chinese Traditional and Herbal Drugs, 50, 2712-2717. (In Chinese)

[39] Chen, Y., Jiang, E., Yan, J. and Tao, Y. (2020) Validation of an Analytical Method Using UPLC-MS/MS to Quantify Four Bioactive Components in Rat Plasma and Its Application to Pharmacokinetic Study of Traditional and Dispensing Granules Decoction of Ziziphi Spinosae Semen. Biomedical Chromatography, 34, e4797. https://doi.org/10.1002/bmc.4797

[40] Shergis, J.L., Ni, X., Sarris, J., Zhang, A.L., Guo, X., Xue, C.C., Lu, C. and Hugel, H. (2017) Ziziphus spinosa Seeds for Insomnia: A Review of Chemistry and Psychopharmacology. Phytomedicine, 34, 38-43. https://doi.org/10.1016/j.phymed.2017.07.004

[41] Li, S., Liu, L., Pan, Y., Ao, H. and Tang, Y. (2018) GC-MS Analysis and Antioxidant Activity Study of Essential Oil from Ligusticum chuanxiong Hort. Chinese Journal of Ethnomedicine and Ethnopharmacy, 27, 16-20. (In Chinese)

[42] Gao, X., Sun, W., Qi, L. and Li, Y. (2018) Ultra-Performance Liquid Chromatography Coupled with Electrospray Ionization/Quadrupole Time-of-Flight Mass Spectrometry for the Rapid Analysis of Constituents in Chuanxiong Rhizoma. Northwest Pharmaceutical Journal, 33, 711-715. (In Chinese)

[43] Yang, J., Feng, X.L., Yu, Y., Wang, Q., Zou, J., Wang, C.X., Mu, Z.Q., Yao, X.S. and Gao, H. (2016) Novel Phthalide Derivatives Identified from Ligusticum chuanxiong (Chuanxiong). Chinese Medicine, 11, Article No. 10. 
https://doi.org/10.1186/s13020-016-0080-2

[44] Li, W., Tang, Y., Chen, Y. and Duan, J.A. (2012) Advances in the Chemical Analysis and Biological Activities of Chuanxiong. Molecules, 17, 10614-10651.

https://doi.org/10.3390/molecules170910614

[45] Zhang, H., Han, T., Yu, C.H., Jiang, Y.P., Peng, C., Ran, X. and Qin, L.P. (2012) Analysis of the Chemical Composition, Acute Toxicity and Skin Sensitivity of Essential Oil from Rhizomes of Ligusticum chuanxiong. Journal of Ethnopharmacology, 144, 791-796. https://doi.org/10.1016/j.jep.2012.10.010

[46] Liu, Q., Zhou, J., Yu, J., Xie, Y., Jiang, X., Yang, H. and Chen, X. (2014) Systematic and Efficient Separation of 11 Compounds from Rhizoma Chuanxiong via Counter-Current Chromatography-Solid Phase Extraction-Counter-Current Chromatography Hyphenation. Journal of Chromatography A, 1364, 204-213.

https://doi.org/10.1016/j.chroma.2014.08.087

[47] Du, J.C., Xie, X.F., Xiong, L., Sun, C. and Peng, C. (2016) Research Progress of Chemical Constituents and Pharmacological Activities of Essential Oil of Ligusticum chuanxiong. China Journal of Chinese Materia Medica, 41, 4328-4333. (In Chinese)

[48] Hu, J., Jia, X., Fang, X., Li, P., He, C. and Chen, M. (2016) Ultrasonic Extraction, Antioxidant and Anticancer Activities of Novel Polysaccharides from Chuanxiong Rhizome. International Journal of Biological Macromolecules, 85, 277-284. https://doi.org/10.1016/j.ijbiomac.2015.12.046

[49] Li, L.J., Su, Y.F. and Yan, S.L. (2016) Three New Phthalide Glycosides from the Rhizomes of Ligusticum chuanxiong. Phytochemistry Letters, 17, 14-17. https://doi.org/10.1016/j.phytol.2016.05.013

[50] Han, W. (2017) Advances in Chemical Constituents and Pharmacological Effects of Ligusticum chuanxiong. Modern Chinese Medicine, 19, 1341-1349. (In Chinese)

[51] Wei, W., Xu, W. and Yang, X.W. (2017) Two New Phthalide Dimers from the Rhizomes of Ligusticum chuanxiong. Journal of Asian Natural Products Research, 19, 704-711. https://doi.org/10.1080/10286020.2016.1275584

[52] Zhang, X., Han, B., Feng, Z., Jiang, J., Yang, Y. and Zhang, P. (2018) Bioactive Thionic Compounds and Aromatic Glycosides from Ligusticum chuanxiong. Acta Pharmaceutica Sinica B, 8, 818-824. https://doi.org/10.1016/j.apsb.2018.04.002

[53] Zhang, X., Han, B., Feng, Z.M., Yang, Y.N., Jiang, J.S. and Zhang, P.C. (2018) Ferulic Acid Derivatives from Ligusticum chuanxiong. Fitoterapia, 125, 147-154. https://doi.org/10.1016/j.fitote.2018.01.005

[54] Liu, J.R., Liu, Y.S., Liu, H.M., Zhang, C.Y., Wei, A.L., Ye, Q. and Guo, L. (2020) Comparison on Volatile Oil Content and Chemical Constituents among Different Parts of Ligusticum chuanxiong and Decoctions with Different Processing Methods. Chinese Journal of Experimental Traditional Medical Formulae, 26, 101-107. (In Chinese) https://doi.org/10.13422/j.cnki.syfjx.20200314

[55] Zhang, L., Zhou, X., Lu, T.T., Mu, C.L., Wang, X.L., Tang, L.J. and Yang, R.P. (2019) Optimization of Extraction Process of Volatile Oil from Ligusticum chuanxiong and GC-MS Analysis of Its Overground Part. Journal of Chinese Medicinal Materials, 42, 607-611. (In Chinese)

[56] Zhang, P., Chen, L., Wang, X., Chen, J., Xu, S., Ye, L. and Yao, Y. (2019) Simultaneous Determination of Night Effective Constituents and Correlation Analysis of Multiconstituents and Antiplatelet Aggregation Bioactivity in Vitro in Chuanxiong Rhizoma Subjected to Different Decoction Times. Journal of Analytical Methods in Chemistry, 2019, Article ID: 8970624. https://doi.org/10.1155/2019/8970624 
[57] Shan, L., Wu, Y., Yuan, L., Zhang, Y., Xu, Y. and Li, Y. (2017) Rapid Screening of Chemical Constituents in Rhizoma Anemarrhenae by UPLC-Q-TOF/MS Combined with Data Postprocessing Techniques. Evidence-Based Complementary and Alternative Medicine, 2017, Article ID: 4032820. https://doi.org/10.1155/2017/4032820

[58] Ji, S., Su, X., Huang, Z., Su, L., Li, L. and Lu, T. (2017) Analysis of Chemical Variations between Crude and Salt-Processed Anemarrhenae rhizoma Using Ultra-High-Performance Liquid Chromatography-Mass Spectrometry Methods. Molecules, 23, 23. https://doi.org/10.3390/molecules23010023

[59] Youn, U.J., Lee, Y.S., Jeong, H., Lee, J., Nam, J.W., Lee, Y.J., Hwang, E.S., Lee, J.H., Lee, D., Kang, S.S. and Seo, E.K. (2009) Identification of Antiadipogenic Constituents of the Rhizomes of Anemarrhena asphodeloides. Journal of Natural Products, 72, 1895-1898. https://doi.org/10.1021/np900397f

[60] Peng, Y., Zhao, L., Lin, D.J., Liu, Y., Zhang, M. and Song, S.J. (2015) The Chemical Constituents' Determination of the Different Processed Products of Anemarrhena asphodeloides Rhizomes by High-Performance Liquid Chromatography Quadrupole Time-of-Flight Mass Spectrometry (HPLC-Q-TOF-MS/MS). Biomedical Chromatography, 30, 508-519. https://doi.org/10.1002/bmc.3575

[61] Bak, J.P., Cho, Y.M., Kim, I., Park, D.W., Kwon, J.E., Jeong, Y.J., Kwak, J.H. and Kang, S.C. (2016) Inhibitory Effects of Norlignans Isolated from Anemarrhena asphodeloides on Degranulation of Rat Basophilic Leukemia-2H3 Cells. Biomedicine \& Pharmacotherapy, 84, 1061-1066. https://doi.org/10.1016/j.biopha.2016.10.039

[62] Nian, S., Liu, E., Fan, Y., Alolga, R.N., Li, H. and Li, P. (2016) Orthogonal Separation Protocol for the Simultaneous Preparation of four Medically Active Compounds from Anemarrhenae Rhizoma by Sequential Polyamide and Macroporous Resin Adsorbent Chromatography. Journal of Separation Science, 39, 3195-3204. https://doi.org/10.1002/jssc.201600274

[63] Sun, Y., Wu, J., Sun, X., Huang, X., Li, L., Liu, Q. and Song, S. (2016) Steroids from the Rhizome of Anemarrhena asphodeloides and Their Cytotoxic Activities. Bioorganic \& Medicinal Chemistry Letters, 26, 3081-3085. https://doi.org/10.1016/j.bmcl.2016.05.012

[64] Wang, F.X., Yuan, J.C., Kang, L.P., Pang, X., Yan, R.Y., Zhao, Y., Zhang, J., Sun, X.G. and Ma, B.P. (2016) An Efficient Approach to Identify Different Chemical Markers between Fibrous Root and Rhizome of Anemarrhena asphodeloides by Ultra High-Performance Liquid Chromatography Quadrupole Time-of-Flight Tandem Mass Spectrometry with Multivariate Statistical Analysis. Journal of Pharmaceutical and Biomedical Analysis, 129, 105-116.

https://doi.org/10.1016/j.jpba.2016.06.038

[65] Yang, B.Y., Zhang, J., Liu, Y. and Kuang, H.X. (2016) Steroidal Saponins from the Rhizomes of Anemarrhena asphodeloides. Molecules, 21, 1075.

https://doi.org/10.3390/molecules21081075

[66] Jia, D., Huang, Z.Y., Fei, C.H., Xue, W.W. and Lu, T.L. (2017) Comprehensive Profiling and Characterization of Chemical Constituents of Rhizome of Anemarrhena asphodeloides Bge. Journal of Chromatography B: Analytical Technologies in the Biomedical and Life Sciences, 1060, 355-366. https://doi.org/10.1016/j.jchromb.2017.06.032

[67] Khang, P.V., Phuong, D.M. and Ma, L. (2017) New Steroids from Anemarrhena asphodeloides Rhizome and Their Alpha-Glucosidase Inhibitory Activity. Journal of Asian Natural Products Research, 19, 468-473.

https://doi.org/10.1080/10286020.2016.1234459 
[68] Sun, X.H., Zhu, F.T., Zhang, Y.W., Chen, F.F., Yu, Y., Song, N.N. and Huang, X.F. (2017) Two New Steroidal Saponins Isolated from Anemarrhena asphodeloides. Chinese Journal of Natural Medicines, 15, 220-224. https://doi.org/10.1016/S1875-5364(17)30038-9

[69] Yang, B.Y., Bi, X.Y., Liu, Y., Li, G.Y., Yin, X. and Kuang, H.X. (2017) Four New Glycosides from the Rhizoma of Anemarrhena asphodeloides. Molecules, 22, 1995. https://doi.org/10.3390/molecules22111995

[70] Han, F.Y., Song, X.Y., Chen, J.J., Yao, G.D. and Song, S.J. (2018) Timosaponin AIII: A Novel Potential Anti-Tumor Compound from Anemarrhena asphodeloides. Steroids, 140, 125-130. https://doi.org/10.1016/j.steroids.2018.09.014

[71] Wang, Z., Cai, J., Fu, Q., Cheng, L., Wu, L., Zhang, W., Zhang, Y., Jin, Y. and Zhang, C. (2018) Anti-Inflammatory Activities of Compounds Isolated from the Rhizome of Anemarrhena asphodeloides. Molecules, 23, 2631. https://doi.org/10.3390/molecules23102631

[72] Li, X., Liu, Y., Guan, W., Xia, Y., Zhou, Y., Yang, B. and Kuang, H. (2019) Physicochemical Properties and Laxative Effects of Polysaccharides from Anemarrhena asphodeloides Bge. in Loperamide-Induced Rats. Journal of Ethnopharmacology, 240, Article ID: 111961. https://doi.org/10.1016/j.jep.2019.111961

[73] Liao, Z.D., Xu, F.Q., Wu, D.L., Zhang, W. and Huang, Q. (2019) A New Benzophenone Isolated from Fibrous Roots of Anemarrhena asphodeloides. China Journal of Chinese Materia Medica, 44, 1392-1396. (In Chinese)

[74] Wu, D.L., Liao, Z.D., Chen, F.F., Zhang, W., Ren, Y.S., Wang, C.C., Chen, X.X., Peng, D.Y. and Kong, L.Y. (2019) Benzophenones from Anemarrhena asphodeloides Bge. Exhibit Anticancer Activity in HepG2 Cells via the NF-kappaB Signaling Pathway. Molecules, 24, 2246. https://doi.org/10.3390/molecules24122246

[75] Zhao, Y.F., Zhou, J., Zhang, M.J., Zhang, M. and Huang, X.F. (2020) Cytotoxic Steroidal Saponins from the Rhizome of Anemarrhena asphodeloides. Steroids, 155, Article ID: 108557. https://doi.org/10.1016/j.steroids.2019.108557

[76] Yang, B., Liu, Z., Hu, J., Lai, X. and Xia, P. (2016) Quantitative Determination of Sarsasapogenin in Rat Plasma Using Liquid Chromatography-Tandem Mass Spectrometry. Journal of Chromatography B: Analytical Technologies in the Biomedical and Life Sciences, 1022, 213-219. https://doi.org/10.1016/j.jchromb.2016.04.020

[77] Zhu, L., Wang, X., Li, S., Qi, E.R., Meng, J., Ching Lam, K.Y., Dong, X., Xu, J., Chen, H. and Zhao, Z. (2020) Qualitative and Quantitative Characterization of Carbohydrate Profiles in Three Different Parts of Poria cocos. Journal of Pharmaceutical and Biomedical Analysis, 179, Article ID: 113009.

https://doi.org/10.1016/j.jpba.2019.113009

[78] Zou, Y.T., Long, F., Wu, C.Y., Zhou, J., Zhang, W., Xu, J.D., Zhang, Y.Q. and Li, S.L. (2019) A Dereplication Strategy for Identifying Triterpene Acid Analogues in Poria cocos by Comparing Predicted and Acquired UPLC-ESI-QTOF-MS/MS Data. Phytochemical Analysis, 30, 292-310. https://doi.org/10.1002/pca.2813

[79] Zhang, G., Wang, H., Xie, W., Wang, Q., Wang, X., Wang, C., Du, Y., Huo, C. and Wang, Q. (2019) Comparison of Triterpene Compounds of Four Botanical Parts from Poria cocos (Schw.) Wolf Using Simultaneous Qualitative and Quantitative Method and Metabolomics Approach. Food Research International, 121, 666-677. https://doi.org/10.1016/j.foodres.2018.12.036

[80] Ling, Y., Chen, M., Wang, K., Sun, Z., Li, Z., Wu, B. and Huang, C. (2012) Systematic Screening and Characterization of the Major Bioactive Components of Poria cocos and Their Metabolites in Rats by LC-ESI-MS ${ }^{n}$. Biomedical Chromatography, 26, 1109-1117. https://doi.org/10.1002/bmc.1756 
[81] Li, S., Wang, Z., Gu, R., Zhao, Y., Huang, W., Wang, Z. and Xiao, W. (2016) A New Epidioxy-Tetracyclic Triterpenoid from Poria cocos Wolf. Natural Product Research, 30, 1712-1717. https://doi.org/10.1080/14786419.2015.1136909

[82] Chen, T., La, H., Chou, G., Mao, X. and Zou, X. (2018) A Unique Naphthone Derivative and a Rare 4,5-Seco-Lanostane Triterpenoid from Poria cocos. Molecules, 23, 2508. https://doi.org/10.3390/molecules23102508

[83] Chen, T., Kan, Y.J., Chou, G.X. and Zhang, C.G. (2018) A New Highly Oxygenated Pregnane and Two New 5-Hydroxymethylfurfural Derivatives from the Water Decoction of Poria cocos. Journal of Asian Natural Products Research, 20, 1101-1107. https://doi.org/10.1080/10286020.2017.1383398

[84] Feng, G.F., Zheng, Y., Sun, Y., Liu, S., Pi, Z.F., Song, F.R. and Liu, Z.Q. (2018) A Targeted Strategy for Analyzing Untargeted Mass Spectral Data to Identify Lanostane-Type Triterpene Acids in Poria cocos by Integrating a Scientific Information System and Liquid Chromatography-Tandem Mass Spectrometry Combined with Ion Mobility Spectrometry. Analytica Chimica Acta, 1033, 87-99. https://doi.org/10.1016/j.aca.2018.06.048

[85] Lee, S., Choi, E., Yang, S.M., Ryoo, R., Moon, E., Kim, S.H. and Kim, K.H. (2018) Bioactive Compounds from Sclerotia Extract of Poria cocos That Control Adipocyte and Osteoblast Differentiation. Bioorganic Chemistry, 81, 27-34.

https://doi.org/10.1016/j.bioorg.2018.07.031

[86] Jin, J., Zhou, R., Xie, J., Ye, H., Liang, X., Zhong, C., Shen, B., Qin, Y., Zhang, S. and Huang, L. (2019) Insights into Triterpene Acids in Fermented Mycelia of Edible Fungus Poria cocos by a Comparative Study. Molecules, 24, 1331

https://doi.org/10.3390/molecules24071331

[87] Li, X., He, Y., Zeng, P., Liu, Y., Zhang, M., Hao, C., Wang, H., Lv, Z. and Zhang, L. (2019) Molecular Basis for Poria cocos Mushroom Polysaccharide Used as an Antitumour Drug in China. Journal of Cellular and Molecular Medicine, 23, 4-20. https://doi.org/10.1111/jcmm.13564

[88] Pu, Y.W., Liu, Z.J., Tian, H. and Bao, Y.X. (2019) The Immunomodulatory Effect of Poria cocos Polysaccharides Is Mediated by the $\mathrm{Ca}^{2+} / \mathrm{PKC} / \mathrm{p} 38 / \mathrm{NF}-\kappa \mathrm{B}$ Signaling Pathway in Macrophages. International Immunopharmacology, 72, 252-257. https://doi.org/10.1016/j.intimp.2019.04.017

[89] Sun, Y. (2014) Biological Activities and Potential Health Benefits of Polysaccharides from Poria cocos and Their Derivatives. International Journal of Biological Macromolecules, 68, 131-134. https://doi.org/10.1016/j.ijbiomac.2014.04.010

[90] Wang, W., Dong, H., Yan, R., Li, H., Li, P., Chen, P., Yang, B. and Wang, Z. (2015) Comparative Study of Lanostane-Type Triterpene Acids in Different Parts of Poria $\operatorname{cocos}$ (Schw.) Wolf by UHPLC-Fourier Transform MS and UHPLC-Triple Quadruple MS. Journal of Pharmaceutical and Biomedical Analysis, 102, 203-214. https://doi.org/10.1016/j.jpba.2014.09.014

[91] Zeng, H., Liu, Q., Yu, J., Jiang, X., Wu, Z., Wang, M., Chen, M. and Chen, X. (2015) One-Step Separation of Nine Structural Analogues from Poria cocos (Schw.) Wolf. via Tandem High-Speed Counter-Current Chromatography. Journal of Chromatography B: Analytical Technologies in the Biomedical and Life Sciences, 1004, 10-16. https://doi.org/10.1016/j.jchromb.2015.09.017

[92] Wang, N., Zhang, Y., Wang, X., Huang, X., Fei, Y., Yu, Y. and Shou, D. (2016) Antioxidant Property of Water-Soluble Polysaccharides from Poria cocos Wolf Using Different Extraction Methods. International Journal of Biological Macromolecules, 83, 103-110. https://doi.org/10.1016/j.ijbiomac.2015.11.032 
[93] Qian, Q., Zhou, N., Qi, P., Zhang, Y., Mu, X., Shi, X. and Wang, Q. (2018) A UHPLC-QTOF-MS/MS Method for the Simultaneous Determination of Eight Triterpene Compounds from Poria cocos (Schw.) Wolf Extract in Rat Plasma: Application to a Comparative Pharmacokinetic Study. Journal of Chromatography B: Analytical Technologies in the Biomedical and Life Sciences, 1102-1103, 34-44. https://doi.org/10.1016/j.jchromb.2018.10.011

[94] Zhang, W., Chen, L., Li, P., Zhao, J. and Duan, J. (2018) Antidepressant and Immunosuppressive Activities of Two Polysaccharides from Poria cocos (Schw.) Wolf. International Journal of Biological Macromolecules, 120, 1696-1704.

https://doi.org/10.1016/j.ijbiomac.2018.09.171

[95] Zhu, L.X., Xu, J., Wang, R.J., Li, H.X., Tan, Y.Z., Chen, H.B., Dong, X.P. and Zhao, Z.Z. (2018) Correlation between Quality and Geographical Origins of Poria cocos Revealed by Qualitative Fingerprint Profiling and Quantitative Determination of Triterpenoid Acids. Molecules, 23, 2200.

https://doi.org/10.3390/molecules23092200

[96] Zhu, L., Xu, J., Zhang, S., Wang, R., Huang, Q., Chen, H., Dong, X. and Zhao, Z. (2018) Qualitatively and Quantitatively Comparing Secondary Metabolites in Three Medicinal Parts Derived from Poria cocos (Schw.) Wolf Using UHPLC-QTOF-MS/MS-Based Chemical Profiling. Journal of Pharmaceutical and Biomedical Analysis, 150, 278-286. https://doi.org/10.1016/j.jpba.2017.11.066

[97] Li, K., Ji, S., Song, W., Kuang, Y., Lin, Y., Tang, S., Cui, Z., Qiao, X., Yu, S. and Ye, M. (2017) Glycybridins A-K, Bioactive Phenolic Compounds from Glycyrrhiza glabra. Journal of Natural Products, 80, 334-346. https://doi.org/10.1021/acs.jnatprod.6b00783

[98] Liu, Y.Y., Yang, Y.N., Feng, Z.M., Jiang, J.S. and Zhang, P.C. (2019) Eight New Triterpenoid Saponins with Antioxidant Activity from the Roots of Glycyrrhiza uralensis Fisch. Fitoterapia, 133, 186-192. https://doi.org/10.1016/j.fitote.2019.01.014

[99] Ji, S., Li, Z., Song, W., Wang, Y., Liang, W., Li, K., Tang, S., Wang, Q., Qiao, X., Zhou, D., Yu, S. and Ye, M. (2016) Bioactive Constituents of Glycyrrhiza uralensis (Licorice): Discovery of the Effective Components of a Traditional Herbal Medicine. Journal of Natural Products, 79, 281-292. https://doi.org/10.1021/acs.jnatprod.5b00877

[100] Asl, M.N. and Hosseinzadeh, H. (2008) Review of Pharmacological Effects of Glycyrrhiza sp. and Its Bioactive Compounds. Phytotherapy Research, 22, 709-724. https://doi.org/10.1002/ptr.2362

[101] Gou, S.H., Liu, J., He, M., Qiang, Y. and Ni, J.M. (2016) Quantification and Bio-Assay of Alpha-Glucosidase Inhibitors from the Roots of Glycyrrhiza uralensis Fisch. Natural Product Research, 30, 2130-2134. https://doi.org/10.1080/14786419.2015.1114940

[102] Chen, J., Li, W.C. and Gu, X.L. (2017) Optimized Extraction, Preliminary Characterization, and in Vitro Antioxidant Activity of Polysaccharides from Glycyrrhiza Uralensis Fisch. Medical Science Monitor, 23, 1783-1791. https://doi.org/10.12659/MSM.900471

[103] Wang, J., Li, J., Wu, X., Liu, S., Li, H. and Gao, W. (2017) Assessment of Genetic Fidelity and Composition: Mixed Elicitors Enhance Triterpenoid and Flavonoid Biosynthesis of Glycyrrhiza uralensis Fisch. Tissue Cultures. Biotechnology and Applied Biochemistry, 64, 211-217. https://doi.org/10.1002/bab.1485

[104] Wang, X.X., Liu, G.Y., Yang, Y.F., Wu, X.W., Xu, W. and Yang, X.W. (2017) Intestinal Absorption of Triterpenoids and Flavonoids from Glycyrrhizae radix et rhi- 
zoma in the Human Caco-2 Monolayer Cell Model. Molecules, 22, 1627. https://doi.org/10.3390/molecules22101627

[105] Wong, Y.F., Cacciola, F., Fermas, S., Riga, S., James, D., Manzin, V., Bonnet, B., Marriott, P.J., Dugo, P. and Mondello, L. (2018) Untargeted Profiling of Glycyrrhiza glabra Extract with Comprehensive Two-Dimensional Liquid Chromatography-Mass Spectrometry Using Multi-Segmented Shift Gradients in the Second Dimension: Expanding the Metabolic Coverage. Electrophoresis, 39, 1993-2000. https://doi.org/10.1002/elps.201700469

[106] Yin, L., Guan, E., Zhang, Y., Shu, Z., Wang, B., Wu, X., Chen, J., Liu, J., Fu, X., Sun, W. and Liu, M. (2018) Chemical Profile and Anti-Inflammatory Activity of Total Flavonoids from Glycyrrhiza uralensis Fisch. Iranian Journal of Pharmaceutical Research, 17, 726-734.

[107] Frattaruolo, L., Carullo, G., Brindisi, M., Mazzotta, S., Bellissimo, L., Rago, V., Curcio, R., Dolce, V., Aiello, F. and Cappello, A.R. (2019) Antioxidant and Anti-Inflammatory Activities of Flavanones from Glycyrrhiza glabra L. (licorice) Leaf Phytocomplexes: Identification of Licoflavanone as a Modulator of NF-kB/MAPK Pathway. Antioxidants (Basel), 8, 186. https://doi.org/10.3390/antiox8060186

[108] Wu, S.C., Yang, Z.Q., Liu, F., Peng, W.J., Qu, S.Q., Li, Q., Song, X.B., Zhu, K. and Shen, J.Z. (2019) Antibacterial Effect and Mode of Action of Flavonoids from Licorice against Methicillin-Resistant Staphylococcus aureus. Frontiers in Microbiology, 10, 2489. https://doi.org/10.3389/fmicb.2019.02489

[109] Wang, C., Chen, L., Cai, Z.C., Chen, C., Liu, Z., Liu, X., Zou, L., Chen, J., Tan, M., Wei, L. and Mei, Y. (2020) Comparative Proteomic Analysis Reveals the Molecular Mechanisms Underlying the Accumulation Difference of Bioactive Constituents in Glycyrrhiza uralensis Fisch under Salt Stress. Journal of Agricultural and Food Chemistry, 68, 1480-1493. https://doi.org/10.1021/acs.jafc.9b04887

[110] Du, Y., He, B., Li, Q., He, J., Wang, D. and Bi, K. (2017) Identification and Analysis of Chemical Constituents and Rat Serum Metabolites in Suan-Zao-Ren Granule Using Ultra High Performance Liquid Chromatography Quadrupole Time-of-Flight Mass Spectrometry Combined with Multiple Data Processing Approaches. Journal of Separation Science, 40, 2914-2924. https://doi.org/10.1002/jssc.201700236

[111] Zhu, Y.X., Chen, L.L., Gong, J.R. and Wang, S.F. (2014) Identification of Constituents in Suanzaoren Tang by LC-Q-TOF-MS and LC-IT-MS. China Journal of Chinese Materia Medica, 39, 457-465. (In Chinese)

[112] Yang, B., Dong, W., Zhang, A., Sun, H., Wu, F., Wang, P. and Wang, X. (2011) Ultra-Performance Liquid Chromatography Coupled with Electrospray Ionization/Quadrupole-Time-of-Flight Mass Spectrometry for Rapid Analysis of Constituents of Suanzaoren Decoction. Journal of Separation Science, 34, 3208-3215. https://doi.org/10.1002/jssc.201100632

[113] Zhu, L., Wang, Z., Zhai, X., Sui, Z., Wang, D., Li, Q., Bi, K., He, B. and Wang, T. (2017) Simultaneous Quantitative Determination of 13 Active Components in the Traditional Chinese Medicinal Preparation Suanzaoren Oral Liquid by HPLC Coupled with Diode Array Detection and Evaporative Light Scattering Detection. Journal of Separation Science, 40, 2320-2325.

https://doi.org/10.1002/jssc.201700061

[114] Kuang, Y., He, B., Du, Y., Li, J., Wang, D., Bi, K. and Li, Q. (2016) Simultaneous Determinations of Eleven Bioactive Components in Suanzaoren Decoction Granules by High-Performance Liquid Chromatography and Its Application to the Quality Control in Productive Processes. Analytical Sciences, 32, 931-936. 
https://doi.org/10.2116/analsci.32.931

[115] Wang, Y., Yang, X., Xia, P.F., Ma, X., Yang, R.Q., Wang, Y.X., Fan, Q. and Zhao, L. (2020) Review of Chemical Constituents, Pharmacological Effects and Clinical Applications of Suanzaoren Decoction and Prediction and Analysis of Its Q-Marker. China Journal of Chinese Materia Medica, 45, 2765-2771. (In Chinese)

[116] Bao, K.D., Li, P., Qi, L.W., Li, H.J., Yi, L., Wang, W. and Wang, Y.Q. (2009) Characterization of Flavonoid Metabolites in Rat Plasma, Urine, and Feces after Oral Administration of Semen Ziziphi Spinosae Extract by HPLC-Diode-Array Detection (DAD) and Ion-Trap Mass Spectrometry (MS). Chemical and Pharmaceutical Bulletin, 57, 144-148. https://doi.org/10.1248/cpb.57.144 